\title{
奇异扰动的二阶非自治格点系统的一致吸引子的 上半连续性
}

雷娜，周盛凡*

浙江师范大学数学与计算机科学学院, 金华 321004

E-mail: 473519681@qq.com, zhoushengfan@yahoo.com

收稿日期: 2021-05-24; 接受日期: 2021-10-08; 网络出版日期: 2021-12-23; * 通信作者

国家自然科学基金 (批准号: 11871437 和 11971356) 资助项目

摘要 本文研究概周期外力驱动的二阶非自治格点系统的一致吸引子在 Hausdorff 半距离下关于二阶 项系数的上半连续性. 首先证明在一定条件下二阶非自治格点系统和对应的一阶非自治格点系统分别 存在一致吸引子. 然后考虑二阶导数项系数趋于零时, 这两个一致吸引子之间的关系. 采用的方法是 在二阶非自治格点系统的相空间中构造一个子集, 使得一阶非自治格点系统的一致吸引子作为第一分 量自然地嵌入到该子集中, 并证明当二阶项系数充分小时, 二阶非自治格点系统的一致吸引子可以进 入到这个子集的任何邻域内. 最后讨论二阶非自治格点系统的一致吸引子在正的二阶导数项系数处的 上半连续性.

关键词 非自治格点系统 奇异扰动一致吸引子 上半连续性

MSC (2020) 主题分类 $37 \mathrm{~L} 50,35 \mathrm{~B} 40,35 \mathrm{Q} 55$

\section{1 引言}

设 $k \in \mathbb{N}$ 以及 Hilbert 空间

$$
\ell^{2}=\left\{u=\left(u_{m}\right)_{m \in \mathbb{Z}^{k}}: m=\left(m_{1}, m_{2}, \ldots, m_{k}\right) \in \mathbb{Z}^{k}, u_{m} \in \mathbb{R}, \sum_{m \in \mathbb{Z}^{k}} u_{m}^{2}<+\infty\right\},
$$

赋予如下常用的内积和范数:

$$
(u, v)=\sum_{m \in \mathbb{Z}^{k}} u_{m} v_{m}, \quad\|u\|^{2}=(u, u), \quad u=\left(u_{m}\right)_{m \in \mathbb{Z}^{k}}, \quad v=\left(v_{m}\right)_{m \in \mathbb{Z}^{k}} \in \ell^{2} .
$$

英文引用格式: Lei N, Zhou S F. Upper semicontinuity of uniform attractors for non-autonomous lattice systems under singular perturbations (in Chinese). Sci Sin Math, 2022, 52: 1121-1144, doi: 10.1360/SCM-2021-0372 
设 $C_{b}\left(\mathbb{R}, \ell^{2}\right)$ 表示从 $\mathbb{R}$ 到 $\ell^{2}$ 的连续有界函数组成的空间, $g_{0}=\left(g_{0, m}\right)_{m \in \mathbb{Z}^{k}}: \mathbb{R} \rightarrow \ell^{2}$ 是 Bohr 意义 下的概周期函数, $\mathcal{H}\left(g_{0}\right)=\overline{\left\{g_{0}(\cdot+h): h \in \mathbb{R}\right\}}{ }^{C_{b}\left(\mathbb{R}, \ell^{2}\right)}\left(C_{b}\left(\mathbb{R}, \ell^{2}\right)\right.$ 中的闭包 $)$. 本文考虑一簇一阶非自治 格点系统

$$
\left\{\begin{array}{l}
\dot{u}_{m}+(A u)_{m}+\lambda_{m} u_{m}+f_{m}\left(u_{j} \mid j \in I_{m q}\right)=g_{m}(t), \quad g=\left(g_{m}\right)_{m \in \mathbb{Z}^{k}} \in \mathcal{H}\left(g_{0}\right), \\
u_{m}(\tau)=u_{\tau, m}, \quad m=\left(m_{1}, m_{2}, \ldots, m_{k}\right) \in \mathbb{Z}^{k}, \quad t>\tau, \quad \tau \in \mathbb{R}
\end{array}\right.
$$

和一簇奇异扰动的二阶非自治格点系统

$$
\left\{\begin{array}{l}
\epsilon \ddot{u}_{m}+\dot{u}_{m}+(A u)_{m}+\lambda_{m} u_{m}+f_{m}\left(u_{j} \mid j \in I_{m q}\right)=g_{m}(t), \quad g=\left(g_{m}\right)_{m \in \mathbb{Z}^{k}} \in \mathcal{H}\left(g_{0}\right), \quad \epsilon>0, \\
u_{m}(\tau)=u_{\tau, m}, \quad \dot{u}_{m}(\tau)=u_{1 \tau, m}, \quad m=\left(m_{1}, m_{2}, \ldots, m_{k}\right) \in \mathbb{Z}^{k}, \quad t>\tau, \quad \tau \in \mathbb{R},
\end{array}\right.
$$

其中, $A$ 是耦合算子, $m \in \mathbb{Z}^{k}, u_{m} \in \mathbb{R}, \lambda_{m}>0, I_{m q}=\left\{j \in \mathbb{Z}^{k}\left|\|j-m\|=\max _{1 \leqslant l \leqslant k}\right| j_{l}-m_{l} \mid \leqslant q\right\}, k, q$ $\in \mathbb{N}, f_{m}\left(u_{j} \mid j \in I_{m q}\right)$ 是非线性函数.

众所周知, 自治和非自治动力系统的渐近行为分别可以用全局吸引子、一致吸引子和拉回吸引子 来描述 ${ }^{[1,2]}$. 自从 2001 年 Bates 等 ${ }^{[3]}$ 研究了一阶格点系统的全局吸引子以来, 关于无穷格点系统的

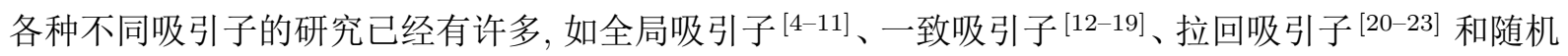
吸引子 [24-27] 等. 现有的文献主要集中在单个一阶或二阶格点系统 (1.1) 和 (1.2) 或它们的耦合系统 的吸引子. 但是一阶和二阶格点系统的吸引子之间的关系是一个有趣的课题. 据我们所知, 对于一阶 和二阶格点系统的吸引子之间的关系的研究, 至今仅有两个文献 $[11,23]$, 它们分别研究了奇异扰动的 二阶自治与非自治格点系统的全局吸引子和拉回吸引子关于 $\epsilon \rightarrow 0^{+}$的上半连续性.

到目前为止, 二阶非自治格点系统 (1.2) 的一致吸引子对于系数 $\epsilon$ 的依赖性, 特别是 (1.2) 与 (1.1) 的一致吸引子之间的关系, 还没有研究.

受文献 $[11,19,23,28]$ 的启发, 本文主要研究奇异扰动的二阶非自治格点系统 (1.2) 的一致吸引子 关于二阶项系数 $\epsilon$ 的上半连续性. 首先考虑 $\epsilon \rightarrow 0^{+}$时, 系统 $(1.2)$ 关于解 $(u(t), \dot{u}(t))$ 的一致吸引子 $\mathcal{A}_{\epsilon}^{\mathcal{H}\left(g_{0}\right)} \subset \ell^{2} \times \ell^{2}$ 和系统 (1.1) 关于解 $u(t)$ 的一致吸引子 $\mathcal{A}^{\mathcal{H}\left(g_{0}\right)} \subset \ell^{2}$ 的关系. 注意到这两个集合位 于不同的空间, 我们应该把它们放在同一空间中去比较. 为此构造一个新子集 $\mathcal{A}_{0}^{\mathcal{H}\left(g_{0}\right)} \subset \ell^{2} \times \ell^{2}$, 使得 $\mathcal{A}^{\mathcal{H}\left(g_{0}\right)}$ 作为第一分量自然嵌入到 $\mathcal{A}_{0}^{\mathcal{H}\left(g_{0}\right)}$ 中, 并证明 $\lim _{\epsilon \rightarrow 0^{+}} d_{h}\left(\mathcal{A}_{\epsilon}^{\mathcal{H}\left(g_{0}\right)}, \mathcal{A}_{0}^{\mathcal{H}\left(g_{0}\right)}\right)=0$, 其中 $d_{h}(\cdot, \cdot)$ 表示 两子集间的 Hausdorff 半距离. 然后证明当 $\epsilon_{0}>0$ 时, $\lim _{\epsilon \rightarrow \epsilon_{0}} d_{h}\left(\mathcal{A}_{\epsilon}^{\mathcal{H}\left(g_{0}\right)}, \mathcal{A}_{\epsilon_{0}}^{\mathcal{H}\left(g_{0}\right)}\right)=0$.

我们知道一个过程的拉回吸引子是相空间中一簇与 $t$ 有关的 “运动” 不变的紧集 $\left\{\mathcal{A}_{\epsilon}(t)\right\}_{t \in \mathbb{R}}$, 而 关于一簇过程的一致吸引子则仅是相空间的一个闭子集, 它与拉回吸引子不同, 一致吸引子对于单个 过程没有不变性. 另一方面, 在已有的文献 $[11,23]$ 中, 关于有限正数 $\epsilon$ 的一个最重要的一致估计式正 是通过全局吸引子和拉回吸引子关于对应的半群或过程的不变性而得到的. 为了获得相似的关于有限 $\epsilon$ 的估计式, 需要借助于一致吸引子 $\mathcal{A}_{\epsilon}^{\mathcal{H}\left(g_{0}\right)}$ 和拉回吸引子 $\left\{\mathcal{A}_{\epsilon}^{g}(t)\right\}_{t \in \mathbb{R}}$ 之间的关系, 并利用 $g \in \mathcal{H}\left(g_{0}\right)$ 是概周期函数 $g_{0}$ 的时间平移函数列的极限函数的特性.

本文的第 2 节引入相空间、给出一些假设并将方程写成向量形式. 第 3 节证明 (1.1) 和 (1.2) 存 在一致吸引子. 第 4 节证明一些一致估计. 第 5 节研究当 $\epsilon \rightarrow 0^{+}$时, $\mathcal{A}_{\epsilon}^{\mathcal{H}\left(g_{0}\right)}$ 的上半连续性. 第 6 节讨 论当 $\epsilon \rightarrow \epsilon_{0}\left(\epsilon_{0}>0\right)$ 时, $\mathcal{A}_{\epsilon}^{\mathcal{H}\left(g_{0}\right)}$ 的上半连续性.

\section{2 预备知识}

本节引入相空间, 给出一些假设并将系统 (1.1) 和 (1.2) 写成向量形式. 
定义 $\ell^{2}$ 上的有界线性算子 $B_{j}$ 和 $\ell^{2}(j=1,2, \ldots, k)$ 分别为

$$
\left(B_{j} u\right)_{m}=\sum_{l=-m_{0}}^{m_{0}} d_{j, l} u_{m_{j l} l}, \quad\left|d_{j, l}\right| \leqslant a_{0} \text { (常数), } \quad u=\left(u_{m}\right)_{m \in \mathbb{Z}^{k}} \in \ell^{2},
$$

其中 $m_{j l}=\left(m_{1}, \ldots, m_{j-1}, m_{j}+l, m_{j+1}, \ldots, m_{k}\right) \in \mathbb{Z}^{k}$. 在 $\ell^{2}$ 上定义如下的双线性内积 $(\cdot, \cdot)_{\lambda}$ 和 $(\cdot, \cdot \cdot)_{\lambda, \epsilon}$ :

$$
\left\{\begin{array}{l}
(u, v)_{\lambda}=\sum_{j=1}^{k}\left(B_{j} u, B_{j} v\right)+(\lambda u, v)=\sum_{j=1}^{k}\left(B_{j} u, B_{j} v\right)+\sum_{m \in \mathbb{Z}^{k}} \lambda_{m} u_{m} v_{m}, \quad u, v \in \ell^{2} . \\
(u, v)_{\lambda, \epsilon}=\epsilon^{-1}(u, v)_{\lambda},
\end{array}\right.
$$

对于 (1.1) 中的 $A 、 \lambda_{m} 、 f_{m}$ 和 $g_{m}$ 作如下的假设:

(A1) 耦合算子 $A$ 是 $\ell^{2}$ 上的自伴非负的线性算子, 具有如下分解:

$$
A=\sum_{j=1}^{k} A_{j}, \quad A_{j}=B_{j}^{*} B_{j}=B_{j} B_{j}^{*}, \quad\left(B_{j} u, v\right)=\left(u, B_{j}^{*} v\right), \quad \forall u, v \in \ell^{2}, \quad j=1, \ldots, k .
$$

(A2) $\forall m \in \mathbb{Z}^{k}, 0<\lambda_{0} \leqslant \lambda_{m} \leqslant \lambda^{0}<+\infty$, 其中 $\lambda_{0}$ 和 $\lambda^{0}$ 是正常数.

(A3) $g_{0}=\left(g_{0, m}\right)_{m \in \mathbb{Z}^{k}}, g_{0}^{\prime}=\left(g_{0, m}^{\prime}\right)_{m \in \mathbb{Z}^{k}}: \mathbb{R} \rightarrow \ell^{2}$ 都是 Bohr 意义下的概周期函数.

(A4) $\forall m \in \mathbb{Z}^{k}, f_{m} \in C^{1}\left(\mathbb{R}^{(2 q+1)^{k}}, \mathbb{R}\right), \quad f_{m}\left(u_{j}=0 \mid j \in I_{m q}\right)=0$, 存在 $\rho \in C\left(\mathbb{R}_{+}, \mathbb{R}_{+}\right)$和 $b=\left(b_{m}\right)_{m \in \mathbb{Z}^{k}} \in \ell^{2}$, 使得

$$
\begin{aligned}
& \sup _{m \in \mathbb{Z}^{k}} \max _{u_{j} \in[-r, r], j \in I_{m q}}\left|f_{m, j}^{\prime}\left(u_{j} \mid j \in I_{m q}\right)\right| \leqslant \rho(r), \\
& f_{m}\left(u_{j} \mid j \in I_{m q}\right) u_{m} \geqslant G_{m}\left(u_{j} \mid j \in I_{m q}\right) \geqslant-b_{m}^{2},
\end{aligned}
$$

其中,

$$
G_{m}\left(u_{j} \mid j \in I_{m q}\right)=\int_{0}^{u_{m}} f_{m}\left(r, u_{j} \mid j \in I_{m q} \backslash\{m\}\right) d r, \quad f_{m, j}^{\prime}\left(u_{j} \mid j \in I_{m q}\right)=\frac{\partial f_{m}}{\partial u_{j}}\left(u_{j} \mid j \in I_{m q}\right),
$$

这里 $f_{m}\left(r, u_{j} \mid j \in I_{m q} \backslash\{m\}\right)$ 是 $f_{m}\left(u_{j} \mid j \in I_{m q}\right)$ 中 $u_{m}$ 用 $r$ 表示的函数.

(A5) $\forall \epsilon>0$, 存在正常数 $\delta_{\epsilon}>0$, 使得

$$
\left|\frac{\partial G_{m}}{\partial u_{j}}\left(u_{j} \mid j \in I_{m q}\right)\right|=\left|G_{m, j}^{\prime}\left(u_{j} \mid j \in I_{m q}\right)\right| \leqslant \delta_{\epsilon}\left|u_{m}\right|, \quad m \neq j, \quad m \in \mathbb{Z}^{k},
$$

其中

$$
0<\delta_{\epsilon}<\min \left\{\frac{\lambda_{0}^{2}}{\sqrt{1+4 \epsilon \lambda_{0}}\left(1+\sqrt{1+4 \epsilon \lambda_{0}}\right)\left[2 \lambda_{0}^{2}(2 q)^{2 k}+1\right]}, \frac{1}{4(2 q)^{2 k}}\right\} .
$$

系统 (1.1) 和 (1.2) 可以写为以下向量形式:

$$
\begin{aligned}
& \dot{u}+A u+\lambda u+f(u)=g(t), \quad u(\tau)=u_{\tau}, \quad t>\tau, \quad g \in \mathcal{H}\left(g_{0}\right), \\
& \epsilon \ddot{u}+\dot{u}+A u+\lambda u+f(u)=g(t), \quad u(\tau)=u_{\tau}, \quad \dot{u}(\tau)=u_{1 \tau}, \quad t>\tau, \quad \epsilon>0, \quad g \in \mathcal{H}\left(g_{0}\right),
\end{aligned}
$$

其中, $u=\left(u_{m}\right)_{m \in \mathbb{Z}^{k}}, \lambda u=\left(\lambda_{m} u_{m}\right)_{m \in \mathbb{Z}^{k}}, f(u)=\left(f_{m}\left(u_{j} \mid j \in I_{m q}\right)\right)_{m \in \mathbb{Z}^{k}}, g(t)=\left(g_{m}(t)\right)_{m \in \mathbb{Z}^{k}}, u(\tau)$ $=\left(u_{m}(\tau)\right)_{m \in \mathbb{Z}^{k}}, u_{1 \tau}=\left(\dot{u}_{m}(\tau)\right)_{m \in \mathbb{Z}^{k}}$. 
对于任意 $g \in \mathcal{H}\left(g_{0}\right), \epsilon>0$, 设 $u_{\epsilon}(\tau)$ 是 $(2.9)$ 的解. 那么问题 (2.9) 可以写成以下形式:

$$
\dot{\psi}_{\epsilon}+H_{\epsilon} \psi_{\epsilon}=F_{\epsilon}\left(\psi_{\epsilon}, g, t\right), \quad \psi_{\epsilon}(\tau)=\left(u_{\epsilon}(\tau), v_{\epsilon}(\tau)\right)^{\mathrm{T}}=\left(u_{\tau}, u_{1 \tau}+\frac{\delta}{\epsilon} u_{\tau}\right)^{\mathrm{T}},
$$

其中,

$$
\begin{aligned}
& \psi_{\epsilon}=\left(\begin{array}{c}
u_{\epsilon} \\
v_{\epsilon}
\end{array}\right), \quad v_{\epsilon}=\dot{u}_{\epsilon}+\frac{\delta}{\epsilon} u_{\epsilon}, \quad \delta=\frac{\epsilon \lambda_{0}}{1+4 \epsilon \lambda_{0}}, \\
& F_{\epsilon}\left(\psi_{\epsilon}, g, t\right)=\left(\begin{array}{c}
0 \\
-\frac{1}{\epsilon} f\left(u_{\epsilon}\right)+\frac{1}{\epsilon} g(t)
\end{array}\right), \\
& H_{\epsilon} \psi_{\epsilon}=\left(\begin{array}{c}
\frac{\delta}{\epsilon} u_{\epsilon}-v_{\epsilon} \\
\frac{1}{\epsilon} A u_{\epsilon}+\frac{1}{\epsilon} \lambda u_{\epsilon}-\frac{\delta}{\epsilon^{2}}(1-\delta) u_{\epsilon}+\frac{1}{\epsilon}(1-\delta) v_{\epsilon}
\end{array}\right) .
\end{aligned}
$$

由 (2.1)、(2.2) 和 (A2) 知, 对于任意 $u \in \ell^{2}$, 有

$$
\lambda_{0}\|u\|^{2} \leqslant \sum_{m \in \mathbb{Z}^{k}} \lambda_{m} u_{m}^{2} \leqslant \sum_{j=1}^{k}\|B u\|^{2}+\sum_{m \in \mathbb{Z}^{k}} \lambda_{m} u_{m}^{2}=\|u\|_{\lambda}^{2} \leqslant\left(a_{0}^{2}\left(2 m_{0}+1\right)^{2} k+\lambda^{0}\right)\|u\|^{2}
$$

和

$$
\epsilon^{-1} \lambda_{0}\|u\|^{2} \leqslant \epsilon^{-1} \sum_{j=1}^{k}\|B u\|^{2}+\epsilon^{-1} \sum_{m \in \mathbb{Z}^{k}} \lambda_{m} u_{m}^{2}=\|u\|_{\lambda, \epsilon}^{2} \leqslant \epsilon^{-1}\left(a_{0}^{2}\left(2 m_{0}+1\right)^{2} k+\lambda^{0}\right)\|u\|^{2} .
$$

从而由 $(\cdot, \cdot)_{\lambda, \epsilon} 、(\cdot, \cdot)_{\lambda}$ 和 $(\cdot, \cdot)$ 诱导的范数 $\|\cdot\|_{\lambda, \epsilon} 、\|\cdot\|_{\lambda}$ 和 $\|\cdot\|$ 是相互等价的. 记

$$
E_{\lambda, \epsilon}=\ell_{\lambda, \epsilon}^{2} \times \ell^{2}, \quad E_{\lambda}=\ell_{\lambda}^{2} \times \ell^{2}, \quad E=\ell^{2} \times \ell^{2},
$$

它们的内积和范数定义如下: 对于 $\psi^{(j)}=\left(u^{(j)}, v^{(j)}\right)^{\mathrm{T}} \in E_{\lambda, \epsilon} 、 \psi^{(j)}=\left(u^{(j)}, v^{(j)}\right)^{\mathrm{T}} \in E_{\lambda}$ 或 $\psi^{(j)}=\left(u^{(j)}\right.$, $\left.v^{(j)}\right)^{\mathrm{T}} \in E(j=1,2)$, 有

$$
\begin{aligned}
& \left(\psi^{(1)}, \psi^{(2)}\right)_{E_{\lambda, \epsilon}}=\left(u^{(1)}, u^{(2)}\right)_{\lambda, \epsilon}+\left(v^{(1)}, v^{(2)}\right), \\
& \left(\psi^{(1)}, \psi^{(2)}\right)_{E_{\lambda}}=\left(u^{(1)}, u^{(2)}\right)_{\lambda}+\left(v^{(1)}, v^{(2)}\right), \\
& \left(\psi^{(1)}, \psi^{(2)}\right)_{E}=\left(u^{(1)}, u^{(2)}\right)+\left(v^{(1)}, v^{(2)}\right) .
\end{aligned}
$$

根据 Bochner-Amerio 定理知, $g_{0}$ 和 $g_{0}^{\prime}$ 的平移函数集 $\left\{g_{0}(\cdot+h)\right\}_{h \in \mathbb{R}}$ 和 $\left\{g_{0}^{\prime}(\cdot+h)\right\}_{h \in \mathbb{R}}$ 在 $C_{b}\left(\mathbb{R}, \ell^{2}\right)^{[2]}$ 中都是预紧的. 因此 $\mathcal{H}\left(g_{0}\right)$ 和 $\mathcal{H}\left(g_{0}^{\prime}\right)=\overline{\left\{g_{0}^{\prime}(\cdot+h): h \in \mathbb{R}\right\}}{ }^{C_{b}\left(\mathbb{R}, \ell^{2}\right)}$ 在 $C_{b}\left(\mathbb{R}, \ell^{2}\right)$ 中是紧的. 设在 $\mathcal{H}\left(g_{0}\right)$ 上的平移算子为

$$
T(h): g \rightarrow T(h) g=g(\cdot+h), \quad \forall g \in \mathcal{H}\left(g_{0}\right), \quad h \in \mathbb{R}_{+},
$$

则

$$
T(h) \mathcal{H}\left(g_{0}\right)=\mathcal{H}\left(g_{0}\right), \quad \forall h \in \mathbb{R}_{+} .
$$


引理 2.1 如果 $(\mathrm{A} 3)$ 成立, 那么对于任意 $g \in \mathcal{H}\left(g_{0}\right)$, 存在序列 $\left\{t_{n}\right\}_{n \in \mathbb{N}} \subset \mathbb{R}$ 使得

$$
g(t)=\lim _{n \rightarrow \infty} g_{0}\left(t+t_{n}\right), \quad g^{\prime}(t)=\lim _{n \rightarrow \infty} g_{0}^{\prime}\left(t+t_{n}\right), \quad \sup _{t \in \mathbb{R}}\left\|g^{\prime}(t)\right\| \leqslant \sup _{t \in \mathbb{R}}\left\|g_{0}^{\prime}(t)\right\| .
$$

证明 由 $(\mathrm{A} 3)$ 、 $\mathcal{H}\left(g_{0}\right)$ 和 $\mathcal{H}\left(g_{0}^{\prime}\right)$ 的定义很容易可以得到 (2.15).

现在介绍过程的拉回吸引子和过程簇的一致吸引子的概念及相关的存在性定理. 设 $X$ 为 Hilbert 空间, $\Sigma$ 是一个参数集或者符号空间.

定义 2.1 设 $U(t, \tau): X \rightarrow X(t \geqslant \tau \in \mathbb{R})$ 是定义在 $X$ 上的双参数映射簇. 如果

(i) $U(t, s) U(s, \tau)=U(t, \tau), \forall t \geqslant s \geqslant \tau$;

(ii) $U(\tau, \tau)=I$ (单位算子), $\forall \tau \in \mathbb{R}$;

(iii) $\forall t \geqslant \tau \in \mathbb{R}, U(t, \tau)$ 在 $X$ 上连续,

则称 $\{U(t, \tau)\}_{t \geqslant \tau}$ 为 $X$ 上的一个连续过程.

如果对于任意 $\sigma \in \Sigma$, 过程 $\left\{U_{\sigma}(t, \tau)\right\}_{t \geqslant \tau}$ 在 $X$ 上是连续的, 其中, $\Sigma$ 是符号空间, $\sigma \in \Sigma$ 是一个符 号, 则 $\left\{U_{\sigma}(t, \tau)\right\}_{t \geqslant \tau, \sigma \in \Sigma}$ 是 $X$ 上的连续过程簇.

定义 2.2 设 $\{U(t, \tau)\}_{t \geqslant \tau}$ 是定义在 $X$ 上的一个连续过程.

(1) 若子集 $D \subset X$ 满足: 对于任意有界集 $B \subset X$, 存在 $t(B)$ 使得对于任意 $t \in \mathbb{R}$, 当 $s \geqslant t(B)$ 时, $U(t, t-s) B \subseteq D$, 则称 $D$ 为 $\{U(t, \tau)\}_{t \geqslant \tau}$ 的拉回吸收集.

(2) 若子集簇 $\{K(t) \subset X\}_{t \in \mathbb{R}}$ 满足:

(i) 紧性: $\forall t \in \mathbb{R}, K(t)$ 是 $X$ 中的紧集;

(ii) 不变性: $U(t, \tau) K(\tau)=K(t), \forall t \geqslant \tau \in \mathbb{R}$;

(iii) 拉回吸引性: 对于任意有界集 $B \subset X, \forall t \in \mathbb{R}, \lim _{\tau \rightarrow-\infty} d_{h}(U(t, \tau) B, K(t))=0$, 则称 $\{K(t)\}_{t \in \mathbb{R}}$ 是 $\{U(t, \tau)\}_{t \geqslant \tau}$ 在 $X$ 上的拉回吸引子.

定义 2.3 若子集 $\mathcal{B}_{0} \subset X$ 满足: 对于任意 $\tau \in \mathbb{R}$ 和每一个有界集 $B \subset X$, 存在 $t \geqslant t(\tau, B)$ 使得 对于任意 $t \geqslant t(\tau, B)$ 有 $\bigcup_{\sigma \in \Sigma} U_{\sigma}(t, \tau) B \subseteq \mathcal{B}_{0}$, 则称 $\mathcal{B}_{0}$ 是连续过程簇 $\left\{U_{\sigma}(t, \tau)\right\}_{t \geqslant \tau, \sigma \in \Sigma}($ 关于 $\sigma \in \Sigma)$ 的一致吸收集.

定义 2.4 如果闭集 $\mathcal{A}_{\Sigma} \subseteq X$ 满足:

(i) 对于任意给定的 $\tau \in \mathbb{R}$ 和任意有界集 $B \subset X, \lim _{t \rightarrow+\infty} \sup _{\sigma \in \Sigma} \operatorname{dist}_{E}\left(U_{\sigma}(t, \tau) B, \mathcal{A}_{\Sigma}\right)=0$;

(ii) $\mathcal{A}_{\Sigma}$ 是具有性质 (i) 的最小闭子集,

则称闭集 $\mathcal{A}_{\Sigma} \subseteq X$ 是过程簇 (关于 $\sigma \in \Sigma$ ) 的一致吸引子.

本文主要研究格点系统 (1.2) 的一致吸引子关于 $\epsilon$ 的上半连续性. 为此, 我们给出定义在无穷序 列空间上的连续过程簇的一致吸引子的存在性定理.

取符号空间为 $\Sigma=\mathcal{H}\left(g_{0}\right)$, 相空间为 $\tilde{X}=\ell^{2}$ 或 $\tilde{X}=\ell^{2} \times \ell^{2}$, 其中 $g_{0}$ 满足 (A3). 根据文献 [18, 定 理 3.1], 可得如下定理.

定理 2.1 设 $\left\{U_{g}(t, \tau)\right\}_{t \geqslant \tau, g \in \mathcal{H}\left(g_{0}\right)}$ 是 $\tilde{X}$ 中的连续过程簇, 满足

$$
U_{g}(t+h, \tau+h)=U_{T(h) g}(t, \tau), \quad \forall g \in \mathcal{H}\left(g_{0}\right), \quad \forall t \geqslant \tau, \quad \tau \in \mathbb{R}, \quad h \in \mathbb{R}_{+} .
$$

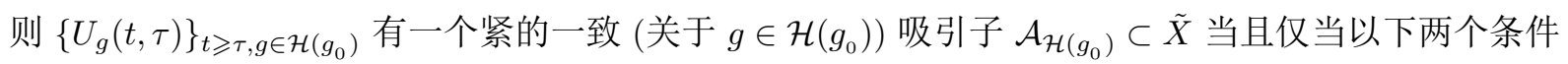
成立:

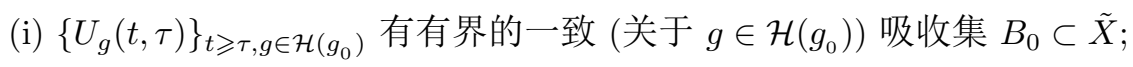


(ii) 对于任意 $\tau \in \mathbb{R}, \varepsilon>0$, 存在 $t_{0}=t_{0}\left(\varepsilon, \tau, B_{0}\right) \geqslant \tau, M\left(\varepsilon, \tau, B_{0}\right) \in \mathbb{N}$, 使得

$$
\sup _{g \in \mathcal{H}\left(g_{0}\right)} \sup _{x \in B_{0}} \sum_{\|m\|>M\left(\varepsilon, \tau, B_{0}\right)}\left|\left(U_{g}(t, \tau) x\right)_{m}\right|^{2} \leqslant \varepsilon^{2}, \quad \forall t \geqslant t_{0}\left(\varepsilon, \tau, B_{0}\right),
$$

其中 $\left|\left(U_{g}(t, \tau) x\right)_{m}\right|$ 是 $\left(U_{g}(t, \tau) x\right)_{m}$ 在 $\mathbb{R}$ 或 $\mathbb{R}^{2}$ 中的范数.

\section{3 一致吸引子的存在性}

本节证明系统 (2.8) 和 (2.10) 存在一致吸引子.

引理 3.1 设条件 (A1)-(A4) 成立, 则有下列结论:

(i) 对于任意 $g \in \mathcal{H}\left(g_{0}\right), \tau \in \mathbb{R}, u(\tau)=u_{\tau} \in \ell^{2}$, 初值问题 (2.8) 有唯一解 $u\left(\cdot, \tau, u_{\tau}\right) \in C\left([\tau,+\infty), \ell^{2}\right)$ $\cap C^{1}\left((\tau,+\infty), \ell^{2}\right)$, 解映射簇

$$
U^{g}(t, \tau): \ell^{2} \ni u(\tau) \rightarrow u\left(t, \tau, u_{\tau}\right)=U^{g}(t, \tau) u(\tau) \in \ell^{2}, \quad t \geqslant \tau, \quad \tau \in \mathbb{R}
$$

生成 $\ell^{2}$ 上的一个连续过程 $\left\{U^{g}(t, \tau)\right\}_{t \geqslant \tau}$ 且满足

$$
U^{g}(t+h, \tau+h)=U^{T(h) g}(t, \tau), \quad \forall g \in \mathcal{H}\left(g_{0}\right), \quad t \geqslant \tau, \quad \tau \in \mathbb{R}, \quad h \in \mathbb{R}_{+} .
$$

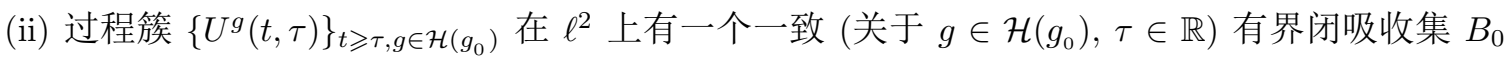
$=B_{0}\left(0, r_{0}\right)=\left\{u \in \ell^{2}:\|u\| \leqslant r_{0}\right\} \subset \ell^{2}$, 其中,

$$
r_{0}=\frac{\sqrt{2\left\|g_{0}\right\|^{2}+4 \lambda_{0}\|b\|^{2}}}{\lambda_{0}}, \quad\left\|g_{0}\right\|^{2}=\sup _{t \in \mathbb{R}}\left\|g_{0}(t)\right\|^{2}=\sup _{t \in \mathbb{R}} \sum_{m \in \mathbb{Z}^{k}} g_{0, m}^{2}(t), \quad\|b\|^{2}=\sum_{m \in \mathbb{Z}^{k}} b_{m}^{2} .
$$

(iii) $\left\{U^{g}(t, \tau)\right\}_{t \geqslant \tau, g \in \mathcal{H}\left(g_{0}\right)}$ 在 $B_{0}$ 上关于 $(g, \tau)$ 是一致拉回渐近零的: 对于任意 $\eta>0$, 存在 $T_{0}\left(\eta, r_{0}\right)$ $>0$ 和 $I_{0}\left(\eta, r_{0}\right) \in \mathbb{N}$, 使得当 $K \geqslant I_{0}\left(\eta, r_{0}\right)$ 和 $s \geqslant T_{0}\left(\eta, r_{0}\right)$ 时, 有

$$
\sup _{\tau \in \mathbb{R}} \sup _{g \in \mathcal{H}\left(g_{0}\right)} \sup _{u(\tau-s) \in B_{0}} \sum_{\|m\| \geqslant 2 K}\left(U^{g}(\tau, \tau-s) u(\tau-s)\right)_{m}^{2} \leqslant \eta \text {. }
$$

(iv) 对于任意 $g \in \mathcal{H}\left(g_{0}\right)$, 连续过程 $\left\{U^{g}(t, \tau)\right\}_{t \geqslant \tau}$ 在 $\ell^{2}$ 上存在唯一的拉回吸引子 $\left\{A^{g}(t) \subseteq B_{0}\right\}_{t \in \mathbb{R}}$, 其中

$$
\mathcal{A}^{g}(t)=\left\{u(t): u(\cdot) \text { 是 }(2.8) \text { 定义于 } \mathbb{R} \text { 上的解且 }\|u(t)\| \leqslant r_{0}, \forall t \in \mathbb{R}\right\} \text {. }
$$

(v) 过程簇 $\left\{U^{g}(t, \tau)\right\}_{t \geqslant \tau, g \in \mathcal{H}\left(g_{0}\right)}$ 在 $\ell^{2}$ 上存在唯一紧的一致吸引子 $A^{\mathcal{H}\left(g_{0}\right)} \subset \ell^{2}$ 且

$$
\mathcal{A}^{\mathcal{H}\left(g_{0}\right)}=\bigcup_{g \in \mathcal{H}\left(g_{0}\right)} \mathcal{A}^{g}(t)=\bigcup_{g \in \mathcal{H}\left(g_{0}\right)} \mathcal{A}^{g}(0) \subseteq B_{0}, \quad \forall t \in \mathbb{R} .
$$

证明 (i) 由 (A3) 可得, 对于任意 $g \in \mathcal{H}\left(g_{0}\right), g$ 在 $\mathbb{R}$ 上是概周期、一致连续和有界的. 此外, $\mathcal{H}(g)=\mathcal{H}\left(g_{0}\right)$. 由 (A4) 得 $f: \ell^{2} \rightarrow \ell^{2}$ 是局部 Lipschitz 的. 因此, 对于任意 $g \in \mathcal{H}\left(g_{0}\right), \tau \in \mathbb{R}$, $u(\tau)=u_{\tau} \in \ell^{2},(2.8)$ 有定义在 $t \in[\tau, \tau+T](T>0)$ 上的唯一解 $u(t)=u\left(t, \tau, u_{\tau}\right)$, 且关于初值 $u_{\tau}$ 是 连续的. 
取内积 $(u(t),(2.8))$, 由 $\|g\|^{2}=\sup _{t \in \mathbb{R}} \sum_{m \in \mathbb{Z}^{k}} g_{m}^{2}(t) \leqslant\left\|g_{0}\right\|^{2}$, 有

$$
\|u(t)\|^{2} \leqslant\|u(\tau)\|^{2} \mathrm{e}^{-\lambda_{0}(t-\tau)}+\frac{1}{\lambda_{0}^{2}}\left\|g_{0}\right\|^{2}+\frac{2}{\lambda_{0}}\|b\|^{2}, \quad \forall \tau \in \mathbb{R}, \quad t \geqslant \tau, \quad g \in \mathcal{H}\left(g_{0}\right) .
$$

因此 $u(\cdot)=u\left(\cdot, \tau, u_{\tau}\right) \in C\left([\tau,+\infty), \ell^{2}\right) \cap C^{1}\left((\tau,+\infty), \ell^{2}\right)$, 解映射簇 $(3.1)$ 生成 $\ell^{2}$ 上的一个连续过程 $\left\{U^{g}(t, \tau)\right\}_{t \geqslant \tau}$ 并且满足 $(3.2)$.

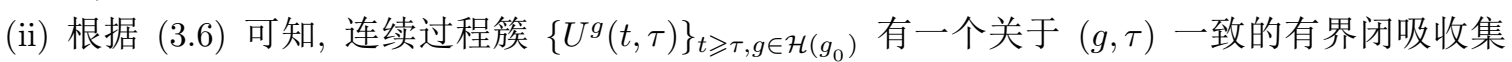
$B_{0}=B_{0}\left(0, r_{0}\right)=\left\{u \in \ell^{2}:\|u\| \leqslant r_{0}\right\} \subset \ell^{2}$, 且存在 $T\left(B_{0}\right) \geqslant 0$ 使得对于任意 $g \in \mathcal{H}\left(g_{0}\right), t \in \mathbb{R}$, $s \geqslant T\left(B_{0}\right)$, 有 $U^{g}(t, t-s) B_{0} \subseteq B_{0}$.

(iii) 选取一个光滑增函数 $\theta \in C^{1}\left(\mathbb{R}_{+},[0,1]\right)$, 满足

$$
\begin{cases}\theta(s)=0, & 0 \leqslant s \leqslant 1, \\ 0 \leqslant \theta(s) \leqslant 1, & 1 \leqslant s \leqslant 2, \\ \theta(s)=1, & s \geqslant 2, \\ \left|\theta^{\prime}(s)\right| \leqslant C_{0}, & s \in \mathbb{R}_{+}, \quad C_{0}>0 .\end{cases}
$$

设 $g \in \mathcal{H}\left(g_{0}\right), \tau \in \mathbb{R}, s \geqslant 0, u_{0} \in B_{0}, u(t)=u(t, \tau-s)=U^{g}(t, \tau-s) u_{0}=\left(u_{m}(t, \tau-s)\right)_{m \in \mathbb{Z}^{k}}(t \geqslant \tau-s)$ 是 $(2.8)$ 的解. 设 $K$ 为正整数, $\|m\|=\max _{1 \leqslant l \leqslant k}\left|m_{l}\right|, m=\left(m_{1}, m_{2}, \ldots, m_{k}\right) \in \mathbb{Z}^{k}, w_{m}=\theta\left(\frac{\|m\|}{K}\right) u_{m}$, $w=\left(w_{m}\right)_{m \in \mathbb{Z}^{k}}$. 取内积 $((2.8), w)$, 由 (ii) 和 (A1)-(A4) 可得, 对于 $s \geqslant T\left(B_{0}\right), t \geqslant \tau \geqslant \tau-s$, 有

$$
\frac{d}{d t} \sum_{m \in \mathbb{Z}^{k}} \theta\left(\frac{\|m\|}{K}\right) u_{m}^{2}(t)+\lambda_{0} \sum_{m \in \mathbb{Z}^{k}} \theta\left(\frac{\|m\|}{K}\right) u_{m}^{2}(t) \leqslant \frac{1}{\lambda_{0}} \sum_{\|m\| \geqslant K} g_{m}^{2}(t)+2 \sum_{\|m\| \geqslant K} b_{m}^{2}+\frac{2 k c_{1}}{K} r_{0}^{2},
$$

其中 $c_{1}=C_{0} m_{0} a_{0}^{2}\left(2 m_{0}+1\right)^{2}$ (不依赖 $\left.K\right)$. 对于任意 $\eta>0$, 存在 $I_{1}\left(\eta, r_{0}\right) \in \mathbb{N}$ 使得

$$
\frac{2 k c_{1}}{K} r_{0}^{2} \leqslant \frac{\eta \lambda_{0}}{6}, \quad \forall K \geqslant I_{1}\left(\eta, r_{0}\right) .
$$

因为 $g_{0}: \mathbb{R} \rightarrow \ell^{2}$ 是概周期的, 所以 $\left\{g_{0}(t): t \in \mathbb{R}\right\}$ 在 $\ell^{2}$ 中是预紧的. 因此存在 $I_{2}\left(\eta, g_{0}\right) \in \mathbb{N}$ 使得

$$
\sup _{t \in \mathbb{R}} \sum_{\|m\| \geqslant K} g_{0, m}^{2}(t) \leqslant \frac{\eta \lambda_{0}}{6}, \quad \forall K \geqslant I_{2}\left(\eta, g_{0}\right) .
$$

结合引理 2.1、(3.10) 以及 $\mathcal{H}\left(g_{0}\right)$ 在 $C_{b}\left(\mathbb{R}, \ell^{2}\right)$ 中的紧性知, 对于 $g \in \mathcal{H}\left(g_{0}\right)$, 存在 $I_{3}\left(\eta, g_{0}\right) \in \mathbb{N}($ 与 $g$ 无 关) 使得

$$
\sup _{t \in \mathbb{R}} \sup _{g \in \mathcal{H}\left(g_{0}\right)} \frac{1}{\lambda_{0}} \sum_{\|m\| \geqslant K} g_{m}^{2}(t) \leqslant \frac{\eta \lambda_{0}}{6}, \quad \forall K \geqslant I_{3}\left(\eta, g_{0}\right) .
$$

由 (A4) 可得, 存在 $I_{4}(\eta) \in \mathbb{N}$ 使得

$$
2 \sum_{\|m\| \geqslant K} b_{m}^{2} \leqslant \frac{\eta \lambda_{0}}{6}, \quad \forall K \geqslant I_{4}(\eta) .
$$

取 $I_{0}\left(\eta, r_{0}\right)=\max \left\{I_{1}\left(\eta, r_{0}\right), I_{3}\left(\eta, g_{0}\right), I_{4}(\eta)\right\}$, 那么由 (3.8)-(3.12) 知, 对于 $K \geqslant I_{0}\left(\eta, r_{0}\right), t \geqslant \tau \geqslant \tau-s$, $\tau \in \mathbb{R}, s \geqslant T\left(B_{0}\right)$, 有

$$
\frac{d}{d t} \sum_{m \in \mathbb{Z}^{k}} \theta\left(\frac{\|m\|}{K}\right) u_{m}^{2}(t)+\lambda_{0} \sum_{m \in \mathbb{Z}^{k}} \theta\left(\frac{\|m\|}{K}\right) u_{m}^{2}(t) \leqslant \frac{\eta \lambda_{0}}{2} .
$$


在 $[\tau-s, \tau]$ 上对 (3.13) 用 Gronwall 不等式, 得

$$
\sum_{m \in \mathbb{Z}^{k}} \theta\left(\frac{\|m\|}{K}\right) u_{m}^{2}(\tau, \tau-s) \leqslant \sum_{m \in \mathbb{Z}^{k}} \theta\left(\frac{\|m\|}{K}\right) u_{m}^{2}(\tau-s) \mathrm{e}^{-\lambda_{0} s}+\frac{\eta}{2} \leqslant r_{0}^{2} \mathrm{e}^{-\lambda_{0} s}+\frac{\eta}{2}, \quad s \geqslant T\left(B_{0}\right) .
$$

设 $T_{0}\left(\eta, r_{0}\right)=\max \left\{T\left(B_{0}\right), \frac{1}{\lambda_{0}} \ln \frac{2 r_{0}^{2}}{\eta}\right\}$, 那么对于 $K \geqslant I_{0}\left(\eta, r_{0}\right), s \geqslant T_{0}\left(\eta, r_{0}\right)$, 有

$$
\sum_{\|m\| \geqslant 2 K} u_{m}^{2}(\tau, \tau-s) \leqslant \sum_{m \in \mathbb{Z}^{k}} \theta\left(\frac{\|m\|}{K}\right) u_{m}^{2}(\tau, \tau-s) \leqslant \eta .
$$

(iv)-(v) 由 (i)-(iii) 和文献 [22, 定理 4.2 和 2.1] 可得证. 证毕.

考虑系统 $(2.10)$, 假设条件 (A5) 成立. 对于 $\epsilon>0$ 以及 $(\mathrm{A} 5)$ 中的 $\delta_{\epsilon}$, 设

$$
\begin{aligned}
& \beta=1-2 \delta_{\epsilon}(2 q)^{2 k} \geqslant \frac{1}{2}>0, \quad \vartheta=\frac{\epsilon \lambda_{0}}{\sqrt{1+4 \epsilon \lambda_{0}}\left(1+\sqrt{1+4 \epsilon \lambda_{0}}\right)} \in(0, \delta), \\
& 0<\delta_{1}=\vartheta-\frac{\delta_{\epsilon} \epsilon\left(2 \lambda_{0}^{2}(2 q)^{2 k}+1\right)}{2 \lambda_{0}}<\vartheta, \quad \mu=\frac{\delta_{1}}{\epsilon}>0 .
\end{aligned}
$$

引理 3.2 对于初值问题 (2.10) 和 $\epsilon>0$, 设条件 (A1)-(A5) 成立, 则有下列结论:

(i) 对于任意 $g \in \mathcal{H}\left(g_{0}\right), \tau \in \mathbb{R}$ 以及 $\psi_{\epsilon}(\tau)=\left(u_{\epsilon}(\tau), v_{\epsilon}(\tau)\right)^{\mathrm{T}} \in E_{\lambda, \epsilon}$, 问题 (2.10) 有唯一解

$$
\psi_{\epsilon}(t)=\psi_{\epsilon}\left(t, \tau, \psi_{\epsilon}(\tau)\right)=\left(u_{\epsilon}\left(t, \tau, \psi_{\epsilon}(\tau)\right), v_{\epsilon}\left(t, \tau, \psi_{\epsilon}(\tau)\right)\right)^{\mathrm{T}} \in C\left([\tau,+\infty), E_{\lambda, \epsilon}\right) \cap C^{1}\left((\tau,+\infty), E_{\lambda, \epsilon}\right),
$$

$\psi_{\epsilon}(t)$ 关于初值 $\psi_{\epsilon}(\tau)$ 连续, 解映射簇

$$
U_{\epsilon}^{g}(t, \tau): E_{\lambda, \epsilon} \ni \psi_{\epsilon}(\tau) \rightarrow \psi_{\epsilon}(t)=\psi_{\epsilon}\left(t, \tau, \psi_{\epsilon}(\tau)\right) \in E_{\lambda, \epsilon}, \quad t \geqslant \tau, \quad \tau \in \mathbb{R}
$$

生成 $E_{\lambda, \epsilon}$ 上的一个连续过程 $\left\{U_{\epsilon}^{g}(t, \tau)\right\}_{t \geqslant \tau}$.

(ii) 过程簇 $\left\{U_{\epsilon}^{g}(t, \tau)\right\}_{t \geqslant \tau, g \in \mathcal{H}\left(g_{0}\right)}$ 在 $E_{\lambda, \epsilon}$ 上有一个一致 (关于 $(g, \tau)$ ) 有界闭吸收集 $B_{\epsilon}=B_{\epsilon}\left(0, r_{\epsilon}\right)$ $=\left\{\psi_{\epsilon} \in E_{\lambda, \epsilon}:\left\|\psi_{\epsilon}\right\|_{E_{\lambda, \epsilon}} \leqslant r_{\epsilon}\right\} \subset E_{\lambda, \epsilon}$, 其中

$$
r_{\epsilon}=\frac{2}{\sqrt{\epsilon}} \sqrt{\frac{1}{\mu}\left\|g_{0}\right\|^{2}+\frac{\lambda_{0}}{\mu}\|b\|^{2}} .
$$

(iii) $\left\{U_{\epsilon}^{g}(t, \tau)\right\}_{t \geqslant \tau, g \in \mathcal{H}\left(g_{0}\right)}$ 在 $B_{\epsilon}$ 上是一致拉回渐近零的: 对于任意 $\eta>0$ 存在 $T_{\epsilon}\left(\eta, r_{\epsilon}\right)>0, I_{\epsilon}\left(\eta, r_{\epsilon}\right)$ $\in \mathbb{N}$ (与 $(g, \tau)$ 无关), 使得对于任意 $\tau \in \mathbb{R}, g \in \mathcal{H}\left(g_{0}\right), K \geqslant I_{\epsilon}\left(\eta, r_{\epsilon}\right), s \geqslant T_{\epsilon}\left(\eta, r_{\epsilon}\right), \psi(\tau-s) \in B_{\epsilon}$, 有

$$
\sum_{\|m\| \geqslant 2 K}\left|\left(U_{\epsilon}^{g}(\tau, \tau-s) \psi(\tau-s)\right)_{m}\right|_{E_{\lambda, \epsilon}}^{2}=\sum_{\|m\| \geqslant 2 K}\left[\epsilon^{-1} \sum_{j=1}^{k}\left(B_{j} u_{\epsilon}\right)_{m}^{2}+\epsilon^{-1} \lambda_{m} u_{\epsilon, m}^{2}+v_{\epsilon, m}^{2}\right](\tau) \leqslant \eta .
$$

(iv) 对于任意 $g \in \mathcal{H}\left(g_{0}\right)$, 连续过程簇 $\left\{U_{\epsilon}^{g}(t, \tau)\right\}_{t \geqslant \tau}$ 在 $E_{\lambda, \epsilon}$ 上有唯一拉回吸引子 $\left\{\mathcal{K}_{\epsilon}^{g}(t) \subseteq B_{\epsilon}\right\}_{t \in \mathbb{R}}$, 其中

$$
\mathcal{K}_{\epsilon}^{g}(t)=\left\{\psi_{\epsilon}(t): \psi_{\epsilon}(\cdot) \text { 是 }(2.10) \text { 的解, }\left\|\psi_{\epsilon}(t)\right\|_{E_{\lambda, \epsilon}} \leqslant r_{\epsilon}, \forall t \in \mathbb{R}\right\} .
$$

(v) $\left\{U^{g}(t, \tau)\right\}_{t \geqslant \tau, g \in \mathcal{H}\left(g_{0}\right)}$ 存在唯一紧的一致吸引子 $\mathcal{K}_{\epsilon}^{\mathcal{H}\left(g_{0}\right)} \subset E_{\lambda, \epsilon}$, 且

$$
\mathcal{K}_{\epsilon}^{\mathcal{H}\left(g_{0}\right)}=\bigcup_{g \in \mathcal{H}\left(g_{0}\right)} \mathcal{K}_{\epsilon}^{g}(t)=\bigcup_{g \in \mathcal{H}\left(g_{0}\right)} \mathcal{K}_{\epsilon}^{g}(0) \subseteq B_{\epsilon} \subset E_{\lambda, \epsilon}, \quad \forall t \in \mathbb{R} .
$$


证明 由 (A3) 和 (A4) 可得 $F_{\epsilon}\left(\psi_{\epsilon}, g, t\right)-H_{\epsilon} \psi_{\epsilon}$ 是从 $E_{\lambda, \epsilon}$ 到 $E_{\lambda, \epsilon}$ 的局部 Lipschitz 映射. 因此, 对于任意 $g \in \mathcal{H}\left(g_{0}\right), \tau \in \mathbb{R}, \psi_{\epsilon}(\tau)=\left(u_{\epsilon}(\tau), v_{\epsilon}(\tau)\right)^{\mathrm{T}} \in E_{\lambda, \epsilon},(2.10)$ 有唯一解

$$
\psi_{\epsilon}(t, \tau)=\left(u_{\epsilon}\left(t, \tau, \psi_{\epsilon}(\tau)\right), v_{\epsilon}\left(t, \tau, \psi_{\epsilon}(\tau)\right)\right)^{\mathrm{T}}, \quad t \in\left[\tau, \tau+T_{1}\right], \quad T_{1}>0,
$$

且 $\psi_{\epsilon}(t, \tau)$ 关于 $\psi_{\epsilon}(\tau)$ 连续. 取内积 $\left((2.10), \psi_{\epsilon}\right)_{E_{\lambda, \epsilon}}$, 则有

$$
\begin{aligned}
& \frac{d}{d t}\left[\left\|\psi_{\epsilon}(t, \tau)\right\|_{E_{\lambda, \epsilon}}^{2}+\frac{2}{\epsilon} \sum_{m \in \mathbb{Z}^{k}}\left(G_{m}\left(u_{\epsilon, j}(t, \tau) \mid j \in I_{m q}\right)+b_{m}^{2}\right)\right] \\
& +\mu\left[\left\|\psi_{\epsilon}(t, \tau)\right\|_{E_{\lambda, \epsilon}}^{2}+\frac{2}{\epsilon} \sum_{m \in \mathbb{Z}^{k}}\left(G_{m}\left(u_{\epsilon, j}(t, \tau) \mid j \in I_{m q}\right)+b_{m}^{2}\right)\right] \\
& \quad \leqslant \frac{1}{\epsilon \beta}\|g\|^{2}+\frac{2 \lambda_{0}}{\epsilon}\|b\|^{2} \leqslant \frac{1}{\epsilon \beta}\left\|g_{0}\right\|^{2}+\frac{2 \lambda_{0}}{\epsilon}\|b\|^{2}
\end{aligned}
$$

和

$$
\left\|\psi_{\epsilon}(t, \tau)\right\|_{E_{\lambda, \epsilon}}^{2} \leqslant\left[\left\|\psi_{\epsilon}(\tau)\right\|_{E_{\lambda, \epsilon}}^{2}+\frac{2}{\epsilon} \sum_{m \in \mathbb{Z}^{k}}\left(G_{m}\left(u_{\epsilon, j}(\tau) \mid j \in I_{m q}\right)+b_{m}^{2}\right)\right] \mathrm{e}^{-\mu(t-\tau)}+\frac{1}{\mu \epsilon \beta}\left\|g_{0}\right\|^{2}+\frac{2 \lambda_{0}}{\mu \epsilon}\|b\|^{2},
$$

其中

$$
\sum_{m \in \mathbb{Z}^{k}} G_{m}\left(u_{\epsilon, j}(\tau) \mid j \in I_{m q}\right) \leqslant \sum_{m \in \mathbb{Z}^{k}} f_{m}\left(u_{\epsilon, j}(\tau) \mid j \in I_{m q}\right) u_{\epsilon, m}(\tau) \leqslant(2 q+1)^{k} \rho\left(\left\|u_{\epsilon}(\tau)\right\|\right)\left\|u_{\epsilon}(\tau)\right\|^{2} .
$$

因此

$$
\left\|\psi_{\epsilon}(t, \tau)\right\|_{E_{\lambda, \epsilon}}^{2} \leqslant\left[\left\|\psi_{\epsilon}(\tau)\right\|_{E_{\lambda, \epsilon}}^{2}+\frac{2(2 q+1)^{k} \rho\left(\left\|u_{\epsilon}(\tau)\right\|\right)\left\|u_{\epsilon}(\tau)\right\|^{2}}{\epsilon}+\frac{2\|b\|^{2}}{\epsilon}\right] \mathrm{e}^{-\mu(t-\tau)}+\frac{r_{\epsilon}^{2}}{2}, \quad t \geqslant \tau .
$$

于是, $\psi_{\epsilon}(\cdot, \tau) \in C^{1}\left([\tau,+\infty), E_{\lambda, \epsilon}\right) \cap C^{1}\left((\tau,+\infty), E_{\lambda, \epsilon}\right)$, 解映射簇 (3.17) 生成 $E_{\lambda, \epsilon}$ 上的一个连续过程 $\left\{U_{\epsilon}^{g}(t, \tau)\right\}_{t \geqslant \tau}$, 且

$$
U_{\epsilon}^{g}(t+h, \tau+h)=U_{\epsilon}^{T(h) g}(t, \tau), \quad \forall g \in \mathcal{H}\left(g_{0}\right), \quad t \geqslant \tau, \quad \tau \in \mathbb{R}, \quad h \in \mathbb{R}_{+} .
$$

(ii) 由 (3.23) 知, 存在 $T\left(B_{\epsilon}\right) \geqslant 0$ 使得对于任意 $t \in \mathbb{R}, s \geqslant T\left(B_{\epsilon}\right)$, 有 $U_{\epsilon}^{g}(t, t-s) B_{\epsilon} \subseteq B_{\epsilon}$.

(iii) 对于给定的 $g \in \mathcal{H}\left(g_{0}\right)$, 设

$$
\psi_{\epsilon}(t, \tau-s)=U_{\epsilon}^{g}(t, \tau-s) \psi_{\epsilon}(\tau-s)=\left(u_{\epsilon}(t, \tau-s), v_{\epsilon}(t, \tau-s)\right)^{\mathrm{T}} \in E_{\lambda, \epsilon}, \quad t \geqslant \tau-s, \quad \tau \in \mathbb{R}, \quad s \geqslant 0
$$

是方程 $(2.10)$ 的解, 且 $\psi_{\epsilon}(\tau-s) \in B_{\epsilon}$.

设 $\theta \in C^{1}\left(\mathbb{R}_{+},[0,1]\right)$ 满足 $(3.7)$. 设 $K \in \mathbb{N}, v_{\epsilon}=\dot{u}_{\epsilon}+\frac{\delta}{\epsilon} u_{\epsilon}, w_{\epsilon, m}=\theta\left(\frac{\|m\|}{K}\right) u_{\epsilon, m}, w_{\epsilon}=\left(w_{\epsilon, m}\right)_{m \in \mathbb{Z}^{k}}$, $z_{\epsilon, m}=\theta\left(\frac{\|m\|}{K}\right) v_{\epsilon, m}, z_{\epsilon}=\left(z_{\epsilon, m}\right)_{m \in \mathbb{Z}^{k}}, y_{\epsilon}=\left(w_{\epsilon}, z_{\epsilon}\right)^{\mathrm{T}}$. 取内积 $\left((2.10), y_{\epsilon}\right)_{E_{\lambda, \epsilon}}$, 则有

$$
\left(\dot{\psi}_{\epsilon}, y_{\epsilon}\right)_{E_{\lambda, \epsilon}}+\left(H_{\epsilon} \psi_{\epsilon}, y_{\epsilon}\right)_{E_{\lambda, \epsilon}}+\left(\frac{1}{\epsilon} f\left(u_{\epsilon}\right), z_{\epsilon}\right)=\left(\frac{1}{\epsilon} g(t), z_{\epsilon}\right), \quad t \geqslant \tau-s .
$$

对于 $t \geqslant \tau \geqslant \tau-s, s \geqslant T\left(B_{\epsilon}\right)$, 有

$$
\left(\dot{\psi}_{\epsilon}, y_{\epsilon}\right)_{E_{\lambda, \epsilon}} \geqslant \frac{1}{2} \frac{d}{d t} \sum_{m \in \mathbb{Z}^{k}} \theta\left(\frac{\|m\|}{K}\right)\left|\psi_{\epsilon, m}\right|_{E_{\lambda, \epsilon}}^{2}-\frac{C_{0} m_{0} a_{0}^{2}\left(2 m_{0}+1\right)^{2} k}{2 \epsilon K} \sum_{m \in Z^{k}}\left(\dot{u}_{\epsilon, m}^{2}+u_{\epsilon, m}^{2}\right)
$$




$$
\begin{aligned}
& \geqslant \frac{1}{2} \frac{d}{d t} \sum_{m \in \mathbb{Z}^{k}} \theta\left(\frac{\|m\|}{K}\right)\left|\psi_{\epsilon, m}\right|_{E_{\lambda, \epsilon}}^{2}-\frac{c_{1} \delta_{2} k}{2 \epsilon K} r_{\epsilon}^{2}, \quad \delta_{2}=2+2 \epsilon \lambda_{0}+\frac{\epsilon}{\lambda_{0}}, \\
& \left(H_{\epsilon} \psi_{\epsilon}, y_{\epsilon}\right)_{E_{\lambda, \epsilon}}=\frac{\delta}{\epsilon^{2}} \sum_{j=1}^{k}\left(B_{j} u_{\epsilon}, B_{j} w_{\epsilon}\right)+\frac{\lambda \delta}{\epsilon^{2}}\left(u_{\epsilon}, w_{\epsilon}\right)+\frac{1}{\epsilon} \sum_{j=1}^{k}\left[\left(B_{j} u_{\epsilon}, B_{j} z_{\epsilon}\right)-\left(B_{j} v_{\epsilon}(t), B_{j} w_{\epsilon}\right)\right] \\
& -\frac{\lambda}{\epsilon}\left(v_{\epsilon}, w_{\epsilon}\right)+\frac{\lambda}{\epsilon}\left(u_{\epsilon}, z_{\epsilon}\right)-\frac{\delta(1-\delta)}{\epsilon^{2}}\left(u_{\epsilon}, z_{\epsilon}\right)+\frac{(1-\delta)}{\epsilon}\left(v_{\epsilon}, z_{\epsilon}\right) \\
& \geqslant-\frac{2 \delta c_{1}}{\epsilon^{2} K} \sum_{m \in Z^{k}} u_{\epsilon, m}^{2}-\frac{c_{1}}{\epsilon K}\left(\sum_{m \in \mathbb{Z}^{k}} u_{\epsilon, m}^{2}+\sum_{m \in \mathbb{Z}^{k}} v_{\epsilon, m}^{2}\right)+\sum_{m \in \mathbb{Z}^{k}} \theta\left(\frac{\|m\|}{K}\right)\left(H_{\epsilon} \psi_{\epsilon}, \psi_{\epsilon}\right)_{E_{\lambda, \epsilon}} \\
& \geqslant-\frac{k c_{1} \delta_{2}(\delta+\epsilon) r_{\epsilon}^{2}}{\epsilon^{2} K}+\sum_{m \in \mathbb{Z}^{k}} \theta\left(\frac{\|m\|}{K}\right)\left(H_{\epsilon} \psi_{\epsilon}, \psi_{\epsilon}\right)_{E_{\lambda, \epsilon}} \\
& \geqslant-\frac{k c_{1} \delta_{2}\left(1+\lambda_{0}\right) r_{\epsilon}^{2}}{\epsilon K}+\sum_{m \in \mathbb{Z}^{k}} \theta\left(\frac{\|m\|}{K}\right)\left(\frac{\vartheta}{\epsilon}\left|\psi_{\epsilon, m}(t)\right|_{E_{\lambda, \epsilon}}^{2}+\frac{1}{2 \epsilon} v_{\epsilon, m}^{2}(t)\right), \\
& \left(\frac{1}{\epsilon} f\left(u_{\epsilon}\right), z_{\epsilon}\right)=\frac{1}{\epsilon} \sum_{m \in \mathbb{Z}^{k}} f_{m}\left(u_{\epsilon, j} \mid j \in I_{m q}\right) \theta\left(\frac{\|m\|}{K}\right) v_{\epsilon, m} \\
& \geqslant \frac{1}{\epsilon} \frac{d}{d t} \sum_{m \in \mathbb{Z}^{k}} \theta\left(\frac{\|m\|}{K}\right) G_{m}\left(u_{\epsilon, j} \mid j \in I_{m q}\right) \\
& +\frac{\delta}{\epsilon^{2}} \sum_{m \in \mathbb{Z}^{k}} \theta\left(\frac{\|m\|}{K}\right) G_{m}\left(u_{\epsilon, j} \mid j \in I_{m q}\right)-\frac{\delta_{\epsilon}(2 q)^{2 k} C_{0} q \delta_{2} r_{\epsilon}^{2}}{2 \epsilon K} \\
& -\frac{\delta_{\epsilon}\left(2 \lambda_{0}^{2}(2 q)^{2 k}+1\right)}{2 \lambda_{0}} \sum_{m \in \mathbb{Z}^{k}} \theta\left(\frac{\|m\|}{K}\right)\left|\psi_{\epsilon, m}\right|_{E_{\lambda, \epsilon}}^{2}-\frac{\delta_{\epsilon}(2 q)^{2 k}}{\epsilon} \sum_{m \in \mathbb{Z}^{k}} \theta\left(\frac{\|m\|}{K}\right) v_{\epsilon, m}^{2}, \\
& \left(\frac{1}{\epsilon} g(t), z\right) \leqslant \frac{1}{2 \epsilon \beta} \sum_{m \in \mathbb{Z}^{k}} \theta\left(\frac{\|m\|}{K}\right) g_{m}^{2}(t)+\frac{\beta}{2 \epsilon} \sum_{m \in \mathbb{Z}^{k}} \theta\left(\frac{\|m\|}{K}\right) v_{\epsilon, m}^{2} .
\end{aligned}
$$

由 (3.25)-(3.29), 得

$$
\begin{aligned}
& \frac{d}{d t}\left[\sum_{m \in \mathbb{Z}^{k}} \theta\left(\frac{\|m\|}{K}\right)\left|\psi_{\epsilon, m}\right|_{E_{\lambda, \epsilon}}^{2}+\frac{2}{\epsilon} \sum_{m \in \mathbb{Z}^{k}} \theta\left(\frac{\|m\|}{K}\right)\left(G_{m}\left(u_{\epsilon, j} \mid j \in I_{m q}\right)+b_{m}^{2}\right)\right] \\
& +\mu\left[\sum_{m \in \mathbb{Z}^{k}} \theta\left(\frac{\|m\|}{K}\right)\left|\psi_{\epsilon, m}\right|_{E_{\lambda, \epsilon}}^{2}+\frac{2}{\epsilon} \sum_{m \in \mathbb{Z}^{k}} \theta\left(\frac{\|m\|}{K}\right)\left(G_{m}\left(u_{\epsilon, j} \mid j \in I_{m q}\right)+b_{m}^{2}\right)\right] \\
& \quad \leqslant \frac{\delta_{3} r_{\epsilon}^{2}}{\epsilon K}+\frac{1}{\epsilon \beta} \sum_{\|m\| \geqslant K} g_{m}^{2}(t)+\frac{2 \lambda_{0}}{\epsilon} \sum_{\|m\| \geqslant K} b_{m}^{2},
\end{aligned}
$$

其中 $\delta_{3}=k c_{1} \delta_{2}\left(3+2 \lambda_{0}\right)+\delta_{\epsilon} \delta_{2}(2 q)^{2 k} C_{0} q$. 类似于 (3.13), 对于任意 $\eta>0$, 存在 $I_{\epsilon}\left(\eta, g_{0}, r_{\epsilon}\right) \in \mathbb{N}($ 与 $(g, \tau)$ 无关) 使得对于 $K \geqslant I_{\epsilon}\left(\eta, g_{0}, r_{\epsilon}\right), t \geqslant \tau \geqslant \tau-s, \tau \in \mathbb{R}, s \geqslant T\left(B_{\epsilon}\right)$, 有

$$
\begin{aligned}
& \frac{d}{d t}\left[\sum_{m \in \mathbb{Z}^{k}} \theta\left(\frac{\|m\|}{K}\right)\left|\psi_{\epsilon, m}(t, \tau-s)\right|_{E_{\lambda, \epsilon}}^{2}+\frac{2}{\epsilon} \sum_{m \in \mathbb{Z}^{k}} \theta\left(\frac{\|m\|}{K}\right)\left(G_{m}\left(u_{\epsilon, j}(t, \tau-s) \mid j \in I_{m q}\right)+b_{m}^{2}\right)\right] \\
& \quad+\mu\left[\sum_{m \in \mathbb{Z}^{k}} \theta\left(\frac{\|m\|}{K}\right)\left|\psi_{\epsilon, m}(t, \tau-s)\right|_{E_{\lambda, \epsilon}}^{2}+\frac{2}{\epsilon} \sum_{m \in \mathbb{Z}^{k}} \theta\left(\frac{\|m\|}{K}\right)\left(G_{m}\left(u_{\epsilon, j}(t, \tau-s) \mid j \in I_{m q}\right)+b_{m}^{2}\right)\right] \\
& \quad \leqslant \frac{\mu \eta}{2}
\end{aligned}
$$


和

$$
\begin{aligned}
& \sum_{m \in \mathbb{Z}^{k}} \theta\left(\frac{\|m\|}{K}\right)\left|\psi_{\epsilon, m}(\tau, \tau-s)\right|_{E_{\lambda, \epsilon}}^{2}+\frac{2}{\epsilon} \sum_{m \in \mathbb{Z}^{k}} \theta\left(\frac{\|m\|}{K}\right)\left(G_{m}\left(u_{\epsilon, j}(\tau, \tau-s) \mid j \in I_{m q}\right)+b_{m}^{2}\right) \\
& \leqslant\left[\sum_{m \in \mathbb{Z}^{k}} \theta\left(\frac{\|m\|}{K}\right)\left|\psi_{\epsilon, m}(\tau-s)\right|_{E_{\lambda, \epsilon}}^{2}+\frac{2}{\epsilon} \sum_{m \in \mathbb{Z}^{k}} \theta\left(\frac{\|m\|}{K}\right)\left(G_{m}\left(u_{\epsilon, j}(\tau-s) \mid j \in I_{m q}\right)+b_{m}^{2}\right)\right] \mathrm{e}^{-\mu s} \\
& \quad+\frac{\eta}{2}
\end{aligned}
$$

其中

$$
\sum_{m \in \mathbb{Z}^{k}} \theta\left(\frac{\|m\|}{K}\right) G_{m}\left(u_{\epsilon, j}(\tau-s) \mid j \in I_{m q}\right) \leqslant \frac{\epsilon(2 q+1)^{k}}{\lambda_{0}} \rho\left(r_{\epsilon} \sqrt{\frac{\epsilon}{\lambda_{0}}}\right) r_{\epsilon}^{2} .
$$

因此

$$
\begin{aligned}
& \sum_{m \in Z^{k}} \theta\left(\frac{\|m\|}{K}\right)\left|\psi_{\epsilon, m}(\tau, \tau-s)\right|_{E_{\lambda, \epsilon}}^{2} \\
& \leqslant\left[r_{\epsilon}^{2}+\frac{2(2 q+1)^{k}}{\lambda_{0}} \rho\left(r_{\epsilon} \sqrt{\frac{\epsilon}{\lambda_{0}}}\right) r_{\epsilon}^{2}+\frac{2}{\epsilon}\|b\|^{2}\right] \mathrm{e}^{-\mu s}+\frac{\eta}{2}, \quad s \geqslant T\left(B_{\epsilon}\right),
\end{aligned}
$$

这蕴涵着 (iii) 成立.

(iv) 和 (v) 由 (i)-(iii) 和文献 [22, 定理 4.2 和 2.1] 可得证. 证毕.

对于任意 $g \in \mathcal{H}\left(g_{0}\right), \tau \in \mathbb{R}, \epsilon>0$, 设 $V_{\epsilon}^{g}(t, \tau)=L_{\epsilon}^{-1} U_{\epsilon}^{g}(t, \tau) L_{\epsilon}$, 其中,

$$
t \geqslant \tau, \quad L_{\epsilon}=\left(\begin{array}{cc}
1 & 0 \\
\frac{\delta}{\epsilon} & 1
\end{array}\right) I:(a, b)^{\mathrm{T}} \rightarrow\left(a, b+\frac{\delta}{\epsilon} a\right)^{\mathrm{T}}
$$

是从 $E$ 到 $E$ 的可逆算子. 根据引理 3.2 可得到以下定理.

定理 3.1 如果条件 $(\mathrm{A} 1)-(\mathrm{A} 5)$ 成立, 那么

(i) 对于任意 $\varphi_{\epsilon}(\tau)=\left(u_{\epsilon}(\tau), \dot{u}_{\epsilon}(\tau)\right)^{\mathrm{T}} \in E,(2.9)$ 有唯一解

$$
\varphi_{\epsilon}(t, \tau)=\varphi_{\epsilon}\left(t, \tau, \varphi_{\epsilon}(\tau)\right)=\left(u_{\epsilon}\left(t, \tau, \varphi_{\epsilon}(\tau)\right), \dot{u}_{\epsilon}\left(t, \tau, \varphi_{\epsilon}(\tau)\right)\right)^{\mathrm{T}} \in C([\tau,+\infty), E) \cap C^{1}((\tau,+\infty), E),
$$

解映射簇

$$
V_{\epsilon}^{g}(t, \tau): E \ni \varphi_{\epsilon}(\tau) \rightarrow \varphi_{\epsilon}(t, \tau)=\varphi_{\epsilon}\left(t, \tau, \varphi_{\epsilon}(\tau)\right) \in E, \quad t \geqslant \tau, \quad \tau \in \mathbb{R}
$$

生成 $E$ 上连续过程 $\left\{V_{\epsilon}^{g}(t, \tau)\right\}_{t \geqslant \tau}, g \in \mathcal{H}\left(g_{0}\right)$.

(ii) 过程簇 $\left\{V_{\epsilon}^{g}(t, \tau)\right\}_{t \geqslant \tau, g \in \mathcal{H}\left(g_{0}\right)}$ 在 $E$ 上有有界闭吸收集 $\tilde{B}_{\epsilon}=\tilde{B}_{\epsilon}\left(0, \tilde{r}_{\epsilon}\right)=\left\{\varphi_{\epsilon} \in E:\left\|\varphi_{\epsilon}\right\|_{E} \leqslant \tilde{r}_{\epsilon}\right\}$ (独立于 $(g, \tau)$ ), 其中

$$
\tilde{r}_{\epsilon}=\frac{2}{\sqrt{\epsilon}} \sqrt{\frac{\delta_{2}}{\mu}\left\|g_{0}\right\|^{2}+\frac{\lambda_{0} \delta_{2}}{\mu}\|b\|^{2}} .
$$

(iii) 对于任意 $\eta>0$, 存在 $\tilde{T}_{\epsilon}\left(\eta, \tilde{r}_{\epsilon}\right)>0, \tilde{I}_{\epsilon}\left(\eta, g_{0}, \tilde{r}_{\epsilon}\right) \in \mathbb{N}$, 使得对于任意 $g \in \mathcal{H}\left(g_{0}\right), \tau \in \mathbb{R}$, $K \geqslant \tilde{I}_{\epsilon}\left(\eta, \tilde{r}_{\epsilon}\right), s \geqslant \tilde{T}_{\epsilon}\left(\eta, \tilde{r}_{\epsilon}\right)$, 有

$$
\sum_{\|m\| \geqslant 2 K}\left\|\varphi_{\epsilon, m}(\tau, \tau-s)\right\|_{E}^{2}=\sum_{\|m\| \geqslant 2 K}\left[u_{\epsilon, m}^{2}(\tau, \tau-s)+\dot{u}_{\epsilon, m}^{2}(\tau, \tau-s)\right] \leqslant \delta_{2} \eta .
$$


(iv) 对于任意 $g \in \mathcal{H}\left(g_{0}\right),\left\{V_{\epsilon}^{g}(t, \tau)\right\}_{t \geqslant \tau}$ 在 $E_{\lambda, \epsilon}$ 上存在唯一拉回吸引子 $\left\{A_{\epsilon}^{g}(t)=L_{\epsilon}^{-1} \mathcal{K}_{\epsilon}^{g}(t) \subseteq \tilde{B}_{\epsilon}\right\}_{t \in \mathbb{R}}$, 其中

$\mathcal{A}_{\epsilon}^{g}(t)=\left\{\left(u_{\epsilon}(t), \dot{u}_{\epsilon}(t)\right): u_{\epsilon}(\cdot)\right.$ 是 $(2.9)$ 定义在 $\mathbb{R}$ 上的解, $\left.\left\|\left(u_{\epsilon}(t), \dot{u}_{\epsilon}(t)\right)\right\|_{E} \leqslant \tilde{r}_{\epsilon}, \forall t \in \mathbb{R}\right\}$.

(v) $\left\{V_{\epsilon}^{g}(t, \tau)\right\}_{t \geqslant \tau, g \in \mathcal{H}\left(g_{0}\right)}$ 存在唯一紧的一致吸引子 $A_{\epsilon}^{\mathcal{H}\left(g_{0}\right)} \subset E$, 且

$$
\mathcal{A}_{\epsilon}^{\mathcal{H}\left(g_{0}\right)}=\bigcup_{g \in \mathcal{H}\left(g_{0}\right)} \mathcal{A}_{\epsilon}^{g}(t)=\bigcup_{g \in \mathcal{H}\left(g_{0}\right)} \mathcal{A}_{\epsilon}^{g}(0)=L_{\epsilon}^{-1} \mathcal{K}_{\epsilon}^{\mathcal{H}\left(g_{0}\right)} \subseteq \tilde{B}_{\epsilon} \subset E, \quad \forall t \in \mathbb{R} .
$$

\section{4 先验估计}

为了研究当 $\epsilon \rightarrow 0^{+}$时 $\mathcal{A}_{\epsilon}^{\mathcal{H}\left(g_{0}\right)}$ 与 $\mathcal{A}^{\mathcal{H}\left(g_{0}\right)}$ 之间的关系. 需要建立一些关于有限 $\epsilon>0$ 的先验估计.

引理 4.1 对于给定的 $\bar{\epsilon}>0$ 和 $\epsilon \in(0, \bar{\epsilon}]$, 假设条件 (A1)-(A5) 成立. 对于任意 $g \in \mathcal{H}\left(g_{0}\right), \tau \in \mathbb{R}$ 和常数 $q_{1} \geqslant 0$, 设 $\varphi_{\epsilon}(t, \tau)=\left(u_{\epsilon}(t, \tau), \dot{u}_{\epsilon}(t, \tau)\right)^{\mathrm{T}}(t \geqslant \tau)$ 是 $(2.9)$ 的解, 其初值满足

$$
\epsilon\left\|\dot{u}_{\epsilon}(\tau)\right\|^{2}+\left\|u_{\epsilon}(\tau)\right\|^{2} \leqslant q_{1} .
$$

则

(i) 存在正常数 $M_{1}=M_{1}(\bar{\epsilon}) 、 \bar{\mu}=\bar{\mu}(\bar{\epsilon}) 、 C_{1}\left(q_{1}\right)$ 和 $\tilde{K}_{1}=\tilde{K}_{1}\left(\bar{\epsilon}, q_{1}\right)>0($ 独立于 $(g, \tau, \epsilon)$, 但依赖于 $\left.g_{0}\right)$, 使得

$$
\begin{aligned}
& \epsilon\left\|\dot{u}_{\epsilon}(t, \tau)\right\|^{2}+\left\|u_{\epsilon}(t, \tau)\right\|^{2} \leqslant M_{1}+C_{1}\left(q_{1}\right) \mathrm{e}^{-\bar{\mu}(t-\tau)}, \quad \forall t \geqslant \tau, \\
& \int_{t}^{t+1}\left\|\dot{u}_{\epsilon}(s)\right\|^{2} d s \leqslant K_{1}\left(\bar{\epsilon}, q_{1}\right), \quad \forall t \geqslant \tau .
\end{aligned}
$$

(ii) 存在正常数 $M_{2}=M_{2}\left(\bar{\epsilon}, q_{1}, \lambda_{0}\right) 、 M_{3}=M_{3}\left(\bar{\epsilon}, q_{1}, \lambda_{0}\right)>0$ (独立于 $(g, \tau, \epsilon)$ ) 以及 $C_{2}\left(q_{1}, \epsilon\right)$ 和 $C_{3}\left(q_{1}, \epsilon\right)>0$ (依赖 $\epsilon$ ), 使得

$$
\begin{aligned}
& \epsilon\left\|\ddot{u}_{\epsilon}(t, \tau)\right\|^{2}+\left\|\dot{u}_{\epsilon}(t, \tau)\right\|^{2} \leqslant M_{2}+C_{2}\left(q_{1}, \epsilon\right) \mathrm{e}^{-\bar{\mu}(t-\tau)}, \quad \forall t \geqslant \tau, \\
& \epsilon\left\|\ddot{u}_{\epsilon}(t, \tau)\right\|^{2}+\left\|\dot{u}_{\epsilon}(t, \tau)\right\|^{2}+\left\|u_{\epsilon}(t, \tau)\right\|^{2} \leqslant M_{3}+C_{3}\left(q_{1}, \epsilon\right) \mathrm{e}^{-\bar{\mu}(t-\tau)}, \quad \forall t \geqslant \tau .
\end{aligned}
$$

证明 对于给定的 $g \in \mathcal{H}\left(g_{0}\right)$, 设 $\varphi_{\epsilon}(t, \tau)=\left(u_{\epsilon}(t, \tau), \dot{u}_{\epsilon}(t, \tau)\right)^{\mathrm{T}}(t \geqslant \tau)$ 是 $(2.9)$ 的解, 且 $\epsilon\left\|\dot{u}_{\epsilon}(\tau)\right\|^{2}$ $+\left\|u_{\epsilon}(\tau)\right\|^{2} \leqslant q_{1}$. 设 $\psi_{\epsilon}(t, \tau)=\left(u_{\epsilon}(t, \tau), v_{\epsilon}(t, \tau)\right)^{\mathrm{T}}$, 其中 $v_{\epsilon}=\dot{u}_{\epsilon}+\frac{\delta}{\epsilon} u_{\epsilon}$. 那么 $\psi_{\epsilon}(t, \tau)$ 是 $(2.10)$ 的解.

由 $\epsilon \in(0, \bar{\epsilon}] 、(\mathrm{~A} 5)$ 和 $(3.16)$, 有

$$
\mu=\frac{\delta_{1}}{\epsilon} \geqslant \frac{\lambda_{0}}{2 \sqrt{1+4 \bar{\epsilon} \lambda_{0}}\left(1+\sqrt{1+4 \bar{\epsilon} \lambda_{0}}\right)} \doteq \bar{\mu}(\bar{\epsilon})=\bar{\mu}>0, \quad \forall \epsilon \in(0, \bar{\epsilon}] .
$$

由 $\frac{\delta^{2}}{\epsilon}=\frac{\epsilon \lambda_{0}^{2}}{\left(1+4 \epsilon \lambda_{0}\right)^{2}}<\frac{\lambda_{0}}{4}$, 有

$$
\epsilon\left\|v_{\epsilon}(t, \tau)\right\|^{2} \geqslant \frac{\epsilon}{2}\left\|\dot{u}_{\epsilon}(t, \tau)\right\|^{2}-\frac{\lambda_{0}}{4}\left\|u_{\epsilon}(t, \tau)\right\|^{2}, \quad \epsilon\left\|v_{\epsilon}(\tau)\right\|^{2} \leqslant 2 \epsilon\left\|\dot{u}_{\epsilon}(\tau)\right\|^{2}+\frac{\lambda_{0}}{2}\left\|u_{\epsilon}(\tau)\right\|^{2} .
$$

由 $(2.11)$ 和 $(3.23)$, 有

$$
\left\|u_{\epsilon}(t, \tau)\right\|^{2}+\epsilon\left\|\dot{u}_{\epsilon}(t, \tau)\right\|^{2} \leqslant\left\|u_{\epsilon}(t, \tau)\right\|_{\lambda}^{2}+\epsilon\left\|v_{\epsilon}(t, \tau)\right\|^{2}
$$




$$
\begin{aligned}
& \leqslant C_{1}\left(q_{1}\right) \mathrm{e}^{-\bar{\mu}(t-\tau)}+M_{0}(\bar{\epsilon}) \\
& \leqslant C_{1}\left(q_{1}\right)+M_{1}, \quad \forall t \geqslant \tau, \quad \tau \in \mathbb{R},
\end{aligned}
$$

其中,

$$
\begin{aligned}
& C_{1}\left(q_{1}\right)=\frac{\left(2+2(2 q+1)^{k} \rho\left(\sqrt{q_{1}}\right)+a_{0}^{2}\left(2 m_{0}+1\right)^{2} k+\lambda^{0}+\frac{\lambda_{0}}{2}\right) q_{1}+2\|b\|^{2}}{\min \left\{\frac{3}{4} \lambda_{0}, \frac{1}{2}\right\}}, \\
& M_{1}=\frac{\frac{2}{\bar{\mu}(\bar{\epsilon})}\left\|g_{0}\right\|^{2}+\frac{2 \lambda_{0}}{\bar{\mu}(\bar{\epsilon})}\|b\|^{2}}{\min \left\{\frac{3}{4} \lambda_{0}, \frac{1}{2}\right\}} .
\end{aligned}
$$

由 (4.7) 和引理 2.1 , 得

$$
\begin{aligned}
\|- & A u_{\epsilon}(t)-\lambda u_{\epsilon}(t)-f\left(u_{\epsilon}(t)\right)+g(t) \|^{2} \\
& \leqslant 4\left[k a_{0}^{4}\left(2 m_{0}+1\right)^{4 k}+\left(\lambda^{0}\right)^{2}+(2 q+1)^{2 k} \rho^{2}\left(\sqrt{C_{1}\left(q_{1}\right)+M_{1}}\right)\right]\left(C_{1}\left(q_{1}\right)+M_{1}\right)+4\left\|g_{0}\right\|^{2} \\
& \doteq K_{2}\left(\bar{\epsilon}, q_{1}\right), \quad t \geqslant \tau .
\end{aligned}
$$

用 $\dot{u}_{\epsilon}$ 与 $(2.9)$ 作内积得

$$
\epsilon \frac{d}{d t}\left\|\dot{u}_{\epsilon}(t)\right\|^{2}+\left\|\dot{u}_{\epsilon}(t)\right\|^{2} \leqslant K_{2}\left(\bar{\epsilon}, q_{1}\right), \quad t \geqslant \tau .
$$

在 $[t, t+1](t \geqslant \tau)$ 上对 (4.7) 两边作积分, 得

$$
\int_{t}^{t+1}\left\|\dot{u}_{\epsilon}(s)\right\|^{2} d s \leqslant K_{2}\left(\bar{\epsilon}, q_{1}\right)+\epsilon\left\|\dot{u}_{\epsilon}(t)\right\|^{2} \leqslant K_{2}\left(\bar{\epsilon}, q_{1}\right)+C_{1}\left(q_{1}\right)+M_{1} \doteq K_{1}\left(\bar{\epsilon}, q_{1}\right), \quad \forall t \geqslant \tau .
$$

(ii) 由 (A3) 和引理 2.1 得 $\left\|g^{\prime}\right\|^{2}=\sup _{t \in \mathbb{R}}\left\|g^{\prime}(t)\right\|^{2} \leqslant\left\|g_{0}^{\prime}\right\|^{2}<\infty$. 令 $\zeta_{\epsilon}(t)=\dot{u}_{\epsilon}(t)$. 对 (2.9) 中的方 程关于 $t$ 求导, 得

$$
\epsilon \ddot{\zeta}_{\epsilon}+\dot{\zeta}_{\epsilon}+A \zeta_{\epsilon}+\lambda \zeta_{\epsilon}+\left(\sum_{j \in I_{m q}} f_{m, j}^{\prime}\left(u_{\epsilon, j} \mid j \in I_{m q}\right) \zeta_{\epsilon, j}\right)_{m \in \mathbb{Z}^{k}}=g^{\prime}(t), \quad t \geqslant \tau,
$$

其中,

$$
\zeta_{\epsilon}(\tau)=\dot{u}_{\epsilon}(\tau), \quad \dot{\zeta}_{\epsilon}(\tau)=\ddot{u}_{\epsilon}(\tau)=\frac{1}{\epsilon}\left(g(\tau)-f\left(u_{\epsilon}(\tau)\right)-\lambda u_{\epsilon}(\tau)-A u_{\epsilon}(\tau)-\dot{u}_{\epsilon}(\tau)\right) .
$$

那么由 (4.1) 得

$$
\begin{aligned}
\left\|\zeta_{\epsilon}(\tau)\right\|^{2}+\epsilon\left\|\dot{\zeta}_{\epsilon}(\tau)\right\|^{2} & \leqslant \frac{5}{\epsilon}\|g\|^{2}+\frac{5}{\epsilon}\left[\frac{1}{5}+(2 q+1)^{2 k} \rho^{2}\left(\sqrt{q_{1}}\right)+\left(\lambda^{0}\right)^{2}+k a_{0}^{4}\left(2 m_{0}+1\right)^{4 k}+\frac{1}{\epsilon}\right] q_{1} \\
& \doteq q_{2}\left(q_{1}, \epsilon\right) .
\end{aligned}
$$

设

$$
\tilde{v}_{\epsilon}=\dot{\zeta}_{\epsilon}+\frac{\delta}{\epsilon} \zeta_{\epsilon}, \quad \tilde{\psi}_{\epsilon}=\left(\zeta_{\epsilon}, \tilde{v}_{\epsilon}\right)^{\mathrm{T}}
$$

类似于 $(3.22)$, 有

$$
\frac{d}{d t}\left\|\tilde{\psi}_{\epsilon}(t)\right\|_{E_{\lambda, \epsilon}}^{2}+\bar{\mu}\left\|\tilde{\psi}_{\epsilon}(t)\right\|_{E_{\lambda, \epsilon}}^{2} \leqslant \frac{2}{\epsilon}\left(\left\|g_{0}^{\prime}\right\|^{2}+(2 q+1)^{2 k} \rho^{2} \sqrt{C_{1}\left(q_{1}\right)+M_{1}}\left\|\dot{u}_{\epsilon}(t)\right\|^{2}\right), \quad \forall t \geqslant \tau .
$$


由 (4.8), 得

$$
\begin{aligned}
\sup _{t \geqslant \tau} & \int_{t}^{t+1} \frac{2}{\epsilon}\left(\left\|g_{0}^{\prime}\right\|^{2}+(2 q+1)^{2 k} \rho^{2} \sqrt{C_{1}\left(q_{1}\right)+M_{1}}\left\|\dot{u}_{\epsilon}(s)\right\|^{2}\right) d s \\
& \leqslant \frac{2}{\epsilon}\left(\left\|g_{0}^{\prime}\right\|^{2}+(2 q+1)^{2 k} \rho^{2} \sqrt{C_{1}\left(q_{1}\right)+M_{1}} K_{1}\left(\bar{\epsilon}, q_{1}\right)\right) \\
& \doteq \frac{1}{\epsilon} K_{3}\left(\bar{\epsilon}, q_{1}\right) .
\end{aligned}
$$

由 (4.10)、(4.11) 和 Gronwall 不等式 (参见文献 [2, 引理 1.3]), 得

$$
\left\|\tilde{\psi}_{\epsilon}(t)\right\|_{E_{\lambda, \epsilon}}^{2} \leqslant\left\|\tilde{\psi}_{\epsilon}(\tau)\right\|_{E_{\lambda, \epsilon}}^{2} \mathrm{e}^{-\bar{\mu}(t-\tau)}+\frac{1}{\epsilon} K_{3}\left(\bar{\epsilon}, q_{1}\right)\left(1+\bar{\mu}^{-1}\right), \quad t \geqslant \tau .
$$

因此,

$$
\epsilon\left\|\ddot{u}_{\epsilon}(t, \tau)\right\|^{2}+\left\|\dot{u}_{\epsilon}(t, \tau)\right\|^{2}=\epsilon\left\|\dot{\zeta}_{\epsilon}(t, \tau)\right\|^{2}+\left\|\zeta_{\epsilon}(t, \tau)\right\|^{2} \leqslant M_{2}+C_{2}\left(q_{1}, \epsilon\right) \mathrm{e}^{-\bar{\mu}(t-\tau)}, \quad t \geqslant \tau,
$$

其中,

$$
\begin{aligned}
& M_{2}=\frac{K_{3}\left(\bar{\epsilon}, q_{1}\right)\left(1+\bar{\mu}^{-1}\right)}{\min \left\{\frac{3}{4} \lambda_{0}, \frac{1}{2}\right\}}, \\
& C_{2}\left(q_{1}, \epsilon\right)=\frac{\left(2+a_{0}^{2}\left(2 m_{0}+1\right)^{2} k+\lambda^{0}+\frac{\lambda_{0}}{2}\right) q_{2}\left(q_{1}, \epsilon\right)}{\min \left\{\frac{3}{4} \lambda_{0}, \frac{1}{2}\right\}} .
\end{aligned}
$$

由 (4.6) 和 (4.12) 可得 (4.5) 成立. 证毕.

引理 4.2 设 $\epsilon \in(0, \bar{\epsilon}](\bar{\epsilon}>0)$ 以及条件 (A1)-(A5) 成立. 对于任意 $g \in \mathcal{H}\left(g_{0}\right), \tau \in \mathbb{R}$, 设 $u_{\epsilon}(t)$ $=u_{\epsilon}(t, \tau)(t \geqslant \tau)$ 是 $(2.9)$ 的解, 且满足

$$
\varphi_{\epsilon}(t)=\left(u_{\epsilon}(t), \dot{u}_{\epsilon}(t)\right)^{\mathrm{T}} \in \mathcal{A}_{\epsilon}^{g}(t) \subseteq \tilde{B}_{\epsilon}, \quad t \in \mathbb{R} .
$$

则

(i) 存在常数 $M_{4}>0$ (独立于 $\left.(g, \epsilon)\right)$, 使得

$$
\epsilon\left\|\ddot{u}_{\epsilon}(t)\right\|^{2}+\left\|\dot{u}_{\epsilon}(t)\right\|^{2}+\left\|u_{\epsilon}(t)\right\|^{2} \leqslant 2 M_{4}, \quad \forall t \in \mathbb{R}, \quad \epsilon \in(0, \bar{\epsilon}] .
$$

(ii) 对于任意 $\eta>0$, 存在 $I_{2}(\eta) \in \mathbb{N}$ (独立于 $(g, \epsilon)$ ), 使得对于 $K \geqslant I_{2}(\eta)$, 有

$$
\sum_{\|m\| \geqslant 2 K}\left|u_{\epsilon, m}(t)\right|^{2} \leqslant \frac{\eta^{2}}{4}, \quad t \in \mathbb{R}, \quad \epsilon \in(0, \bar{\epsilon}] .
$$

(iii) 对于任意 $\tilde{\varphi}_{\epsilon}=\left(u_{\epsilon}, \tilde{w}_{\epsilon}\right)^{\mathrm{T}}=\left(\left(u_{\epsilon, m}\right)_{m \in \mathbb{Z}^{k}},\left(\tilde{w}_{\epsilon, m}\right)_{m \in \mathbb{Z}^{k}}\right)^{\mathrm{T}} \in A_{\epsilon}^{\mathcal{H}\left(g_{0}\right)}$, 有

$$
\left\|\tilde{\varphi}_{\epsilon}\right\|_{E}^{2}=\left\|u_{\epsilon}\right\|^{2}+\left\|\tilde{w}_{\epsilon}\right\|^{2} \leqslant 2 M_{4}, \quad \forall \epsilon \in(0, \bar{\epsilon}]
$$

以及对于任意 $\eta>0$, 有

$$
\sum_{\|m\| \geqslant 2 K} u_{\epsilon, m}^{2} \leqslant \frac{\eta^{2}}{4}, \quad \forall K \geqslant I_{2}(\eta), \quad \epsilon \in(0, \bar{\epsilon}] .
$$


证明 (i) 因为 $V_{\epsilon}^{g}(t, \tau) \mathcal{A}_{\epsilon}^{g}(\tau)=\mathcal{A}_{\epsilon}^{g}(t), \forall t \geqslant \tau$, 所以存在 $\varphi_{\epsilon}(\tau)=\left(u_{\epsilon}(\tau), \dot{u}_{\epsilon}(\tau)\right)^{\mathrm{T}} \in \mathcal{A}_{\epsilon}^{g}(\tau) \subseteq \tilde{B}_{\epsilon}$, 使 得 $\varphi_{\epsilon}(t)=V_{\epsilon}^{g}(t, \tau) \varphi_{\epsilon}(\tau)$ 以及

$$
\epsilon\left\|\dot{u}_{\epsilon}(\tau)\right\|^{2}+\left\|u_{\epsilon}(\tau)\right\|^{2} \leqslant \frac{\epsilon}{\min \left\{\frac{3}{4} \lambda_{0}, \frac{1}{2}\right\}}\left\|\psi_{\epsilon}(\tau)\right\|_{E_{\lambda, \epsilon}}^{2} \leqslant \frac{4 \tilde{\delta}_{2}\left\|g_{0}\right\|^{2}+4 \lambda_{0} \tilde{\delta}_{2}\|b\|^{2}}{\bar{\mu} \min \left\{\frac{3}{4} \lambda_{0}, \frac{1}{2}\right\}} \doteq q_{4},
$$

其中 $\tilde{\delta}_{2}=2+2 \bar{\epsilon} \lambda_{0}+\frac{\bar{\epsilon}}{\lambda_{0}}$. 由引理 4.1(ii) 知, 存在正常数 $M_{4}=M_{4}(\bar{\epsilon})>0 、 \bar{\mu}=\bar{\mu}(\bar{\epsilon})>0($ 独立于 $(g, \epsilon))$ 和 $C_{4}\left(q_{4}, \epsilon\right)$ (依赖于 $\epsilon$ ), 使得

$$
\epsilon\left\|\ddot{u}_{\epsilon}(t)\right\|^{2}+\left\|\dot{u}_{\epsilon}(t)\right\|^{2}+\left\|u_{\epsilon}(t)\right\|^{2} \leqslant M_{4}+C_{4}\left(q_{4}, \epsilon\right) \mathrm{e}^{-\bar{\mu}(t-\tau)}, \quad t \geqslant \tau, \quad \epsilon \in(0, \bar{\epsilon}] .
$$

因此, 对于给定的 $t \in \mathbb{R}$ 和 $\epsilon \in(0, \bar{\epsilon}]$, 总可取 $\tau_{\epsilon} \leqslant t$ 使得 $C_{4}\left(q_{4}, \epsilon\right) \mathrm{e}^{-\bar{\mu}\left(t-\tau_{\epsilon}\right)} \leqslant M_{4}$, 从而有

$$
\epsilon\left\|\ddot{u}_{\epsilon}(t)\right\|^{2}+\left\|\dot{u}_{\epsilon}(t)\right\|^{2}+\left\|u_{\epsilon}(t)\right\|^{2} \leqslant 2 M_{4}, \quad \forall t \in \mathbb{R}, \quad \epsilon \in(0, \bar{\epsilon}],
$$

这蕴涵着 $(4.14)$ 成立.

(ii) 令 $\psi_{\epsilon}(t)=\psi_{\epsilon}(t, t-s)=\left(u_{\epsilon}(t), v_{\epsilon}(t)\right)^{\mathrm{T}}=\left(u_{\epsilon}(t), \dot{u}_{\epsilon}(t)+\frac{\delta}{\epsilon} u_{\epsilon}(t)\right)^{\mathrm{T}}, t \in \mathbb{R}, s \geqslant 0$, 那么 $\psi_{\epsilon}(t) \in \mathcal{K}_{\epsilon}^{g}(t)$ $\subseteq B_{\epsilon}$ 是 (2.10) 的解. 由 (4.14) 和 (3.26)-(3.30), 有

$$
\begin{aligned}
& \frac{d}{d t}\left[\sum_{m \in \mathbb{Z}^{k}} \theta\left(\frac{\|m\|}{K}\right)\left|\psi_{\epsilon, m}\right|_{E_{\lambda, \epsilon}}^{2}+\frac{2}{\epsilon} \sum_{m \in \mathbb{Z}^{k}} \theta\left(\frac{\|m\|}{K}\right)\left(G_{m}\left(u_{\epsilon, j} \mid j \in I_{m q}\right)+b_{m}^{2}\right)\right] \\
& +\mu\left[\sum_{m \in \mathbb{Z}^{k}} \theta\left(\frac{\|m\|}{K}\right)\left|\psi_{\epsilon, m}\right|_{E_{\lambda, \epsilon}}^{2}+\frac{2}{\epsilon} \sum_{m \in \mathbb{Z}^{k}} \theta\left(\frac{\|m\|}{K}\right)\left(G_{m}\left(u_{\epsilon, j} \mid j \in I_{m q}\right)+b_{m}^{2}\right)\right] \\
& \quad \leqslant \frac{1}{\epsilon}\left(\frac{\delta_{4}}{K}+2 \sum_{\|m\| \geqslant K} g_{m}^{2}(t)+2 \lambda_{0} \sum_{\|m\| \geqslant K} b_{m}^{2}\right),
\end{aligned}
$$

其中 $\delta_{4}=2 k c_{1} M_{4}\left(8+2 \lambda_{0}+4 \lambda_{0}^{2}\right)+\frac{C_{0} q M_{4}}{2}$ (与 $(g, \epsilon)$ 无关). 由 (3.9)、(3.11)、(3.12) 以及 $\mu \geqslant \bar{\mu}$ 知, 对 于任意 $\eta>0$, 存在 $I_{2}(\eta) \in \mathbb{N}$ (不依赖于 $(g, \epsilon)$ ), 使得对于 $K \geqslant I_{2}(\eta), t \in \mathbb{R}, s \geqslant 0$, 有

$$
\begin{aligned}
& \frac{d}{d t}\left[\sum_{m \in \mathbb{Z}^{k}} \theta\left(\frac{\|m\|}{K}\right)\left|\psi_{\epsilon, m}(t)\right|_{E_{\lambda, \epsilon}}^{2}+\frac{2}{\epsilon} \sum_{m \in \mathbb{Z}^{k}} \theta\left(\frac{\|m\|}{K}\right)\left(G_{m}\left(u_{\epsilon, j}(t) \mid j \in I_{m q}\right)+b_{m}^{2}\right)\right] \\
& +\bar{\mu}\left[\sum_{m \in \mathbb{Z}^{k}} \theta\left(\frac{\|m\|}{K}\right)\left|\psi_{\epsilon, m}(t)\right|_{E_{\lambda, \epsilon}}^{2}+\frac{2}{\epsilon} \sum_{m \in \mathbb{Z}^{k}} \theta\left(\frac{\|m\|}{K}\right)\left(G_{m}\left(u_{\epsilon, j}(t) \mid j \in I_{m q}\right)+b_{m}^{2}\right)\right] \\
& \quad \leqslant \frac{\bar{\mu} \lambda_{0} \eta^{2}}{8 \epsilon} .
\end{aligned}
$$

因为 $\psi_{\epsilon}(t-s) \in \mathcal{K}_{\epsilon}^{g}(t-s) \subseteq B_{\epsilon},\left(u_{\epsilon}(t-s), \dot{u}_{\epsilon}(t-s)\right)^{\mathrm{T}} \in \mathcal{A}_{\epsilon}^{g}(t-s) \subseteq \tilde{B}_{\epsilon}$, 所以有

$$
\sum_{m \in \mathbb{Z}^{k}} \theta\left(\frac{\|m\|}{K}\right) G_{m}\left(u_{\epsilon, m}(t-s) \mid j \in I_{m q}\right) \leqslant 2(2 q+1)^{k} \rho\left(\sqrt{2 M_{4}}\right) M_{4} .
$$

由 (i) 和 (4.14), 得

$$
\left\|\psi_{\epsilon}(t-s)\right\|^{2} \leqslant \frac{2 M_{4}\left(a_{0}^{2}\left(2 m_{0}+1\right)^{2} k+\lambda^{0}+\frac{\lambda_{0}}{2}+2 \bar{\epsilon}\right)}{\epsilon} \doteq \frac{r}{\epsilon},
$$

其中 $r$ 独立于 $(g, \epsilon)$. 在 $[t-s, t]$ 上对 (4.19) 用 Gronwall 不等式, 有

$$
\sum_{m \in \mathbb{Z}^{k}} \theta\left(\frac{\|m\|}{K}\right)\left|\psi_{\epsilon, m}(t, t-s)\right|_{E_{\lambda, \epsilon}}^{2}
$$




$$
\begin{aligned}
& \leqslant\left[\sum_{m \in \mathbb{Z}^{k}} \theta\left(\frac{\|m\|}{K}\right)\left|\psi_{\epsilon, m}(t-s)\right|_{E_{\lambda, \epsilon}}^{2}+\frac{2}{\epsilon} \sum_{m \in \mathbb{Z}^{k}} \theta\left(\frac{\|m\|}{K}\right)\left(G_{m}\left(u_{\epsilon, j}(t-s) \mid j \in I_{m q}\right)+b_{m}^{2}\right)\right] \mathrm{e}^{-\bar{\mu} s}+\frac{\lambda_{0} \eta^{2}}{8 \epsilon} \\
& \leqslant\left[\frac{r+4 M_{4}(2 q+1)^{k} \rho \sqrt{2 M_{4}}+2\|b\|^{2}}{\epsilon}\right] \mathrm{e}^{-\bar{\mu} s}+\frac{\lambda_{0} \eta^{2}}{8 \epsilon} .
\end{aligned}
$$

于是, 存在 $T_{2}(\eta) \geqslant 0$ (独立于 $(g, \epsilon)$ ), 使得对于 $K \geqslant I_{2}(\eta)$ 和 $s \geqslant T_{2}(\eta)$, 有

$$
\sum_{\|m\| \geqslant 2 K}\left(\epsilon^{-1} \sum_{j=1}^{k}\left(B_{j} u\right)_{m}^{2}+\epsilon^{-1} \lambda_{m} u_{m}^{2}+v_{m}^{2}\right)(t, t-s) \leqslant \sum_{\|m\| \geqslant 2 K}\left|\psi_{\epsilon, m}(t, t-s)\right|_{E_{\lambda, \epsilon}}^{2} \leqslant \frac{\lambda_{0} \eta^{2}}{4 \epsilon},
$$

这蕴涵着 $(4.15)$ 成立.

(iii) 根据定理 3.1 得 $\mathcal{A}_{\epsilon}^{\mathcal{H}\left(g_{0}\right)}=\bigcup_{g \in \mathcal{H}\left(g_{0}\right)} \mathcal{A}_{\epsilon}^{g}(0) \subseteq \tilde{B}_{\epsilon} \subset E$. 对于

$$
\tilde{\varphi}=(u, \tilde{w})^{\mathrm{T}}=\left(\left(u_{m}\right)_{m \in \mathbb{Z}^{k}},\left(\tilde{w}_{m}\right)_{m \in \mathbb{Z}^{k}}\right)^{\mathrm{T}} \in \mathcal{A}_{\epsilon}^{\mathcal{H}\left(g_{0}\right)},
$$

必存在 $g \in \mathcal{H}\left(g_{0}\right)$, 使得 $\tilde{\varphi} \in \mathcal{A}_{\epsilon}^{g}(0)$. 因此, (i)-(ii) 蕴涵 (iii) 成立. 证毕.

\section{5 一致吸引子关于二阶项系数在零点处的上半连续性}

本节研究当 $\epsilon \rightarrow 0^{+}$时系统 $(2.9)$ 的一致吸引子 $\mathcal{A}_{\epsilon}^{\mathcal{H}\left(g_{0}\right)} \subset \ell^{2} \times \ell^{2}=E$ 的上半连续性. 当 $\epsilon=0$ 时, (2.9) 退化为一阶格点系统 $(2.8)$, 并且存在一致吸引子 $\mathcal{A}^{\mathcal{H}\left(g_{0}\right)} \subset \ell^{2}$. 为了比较 $\mathcal{A}_{\epsilon}^{\mathcal{H}\left(g_{0}\right)}$ 与 $\mathcal{A}^{\mathcal{H}\left(g_{0}\right)}$ 之间 的关系, 我们应该把它们放在同一个空间 $\ell^{2} \times \ell^{2}$ 中. 为此, 根据 $\mathcal{A}_{\epsilon}^{\mathcal{H}\left(g_{0}\right)}$ 和 $\mathcal{A}^{\mathcal{H}\left(g_{0}\right)}$ 的结构, 设

$$
\mathcal{A}_{0}^{g}(t)=\left\{(u, \omega) \mid u \in \mathcal{A}^{g}(t), \omega=-A u-\lambda u-f(u)+g(t)\right\} \subset E, \quad t \in \mathbb{R}, \quad g \in \mathcal{H}\left(g_{0}\right)
$$

以及

$$
\mathcal{A}_{0}^{\mathcal{H}\left(g_{0}\right)}=\bigcup_{g \in \mathcal{H}\left(g_{0}\right)} \mathcal{A}_{0}^{g}(0) \subset E .
$$

当 $t \in \mathbb{R}$ 固定时, 根据 $\mathcal{A}^{g}(t)$ 的紧性和 $-A u-\lambda u-f(u)+g(t)$ 关于 $u$ 的连续性, 可知 $\mathcal{A}_{0}^{g}(t)$ 在 $E$ 中 是紧的. 从 (3.5)、(5.1) 和 (5.2) 中可以容易看出, $\mathcal{A}^{\mathcal{H}\left(g_{0}\right)}$ 作为第一分量自然地嵌入到 $\mathcal{A}_{0}^{\mathcal{H}\left(g_{0}\right)}$ 中, 即 $\Pi_{1} \mathcal{A}_{0}^{\mathcal{H}\left(g_{0}\right)}=\mathcal{A}^{\mathcal{H}\left(g_{0}\right)}$, 其中 $\Pi_{1}:(u, \omega) \in \ell^{2} \times \ell^{2} \rightarrow u \in \ell^{2}$ 是从 $\ell^{2} \times \ell^{2}$ 到 $\ell^{2}$ 的投影. 下面证明如下的上 半连续性:

$$
\lim _{\epsilon \rightarrow 0^{+}} d_{h}\left(\mathcal{A}_{\epsilon}^{\mathcal{H}\left(g_{0}\right)}, \mathcal{A}_{0}^{\mathcal{H}\left(g_{0}\right)}\right)=0 .
$$

引理 5.1 设 $\epsilon \in(0, \bar{\epsilon}](\bar{\epsilon}>0)$ 以及条件 $(\mathrm{A} 1)-(\mathrm{A} 5)$ 成立. 任取正实数列 $\left\{\epsilon_{n}\right\}_{n=1}^{+\infty} \subset(0, \bar{\epsilon}]$ 满足: 当 $n \rightarrow+\infty$ 时, $\epsilon_{n} \rightarrow 0$. 取 $\varphi^{(n)}=\left(u^{(n)}, \omega^{(n)}\right)^{\mathrm{T}} \in A_{\epsilon_{n}}^{\mathcal{H}\left(g_{0}\right)}$, 那么存在 $\{n\}$ 的子列 $\left\{k_{k}\right\}$, 使得

$$
\left(u^{\left(k_{k}\right)}, \omega^{\left(k_{k}\right)}\right)^{\mathrm{T}} \rightarrow(\bar{u}, \tilde{u})^{\mathrm{T}} \in \mathcal{A}_{0}^{\mathcal{H}\left(g_{0}\right)}(k \rightarrow+\infty) \text { 在 } E \text { 中强收敛. }
$$

证明 对于任意 $n \in \mathbb{N}$, 取 $\varphi_{0}^{(n)}=\left(u_{0}^{(n)}, \omega_{0}^{(n)}\right)^{\mathrm{T}} \in \mathcal{A}_{\epsilon_{n}}^{\mathcal{H}\left(g_{0}\right)}=\bigcup_{g \in \mathcal{H}\left(g_{0}\right)} \mathcal{A}_{\epsilon_{n}}^{g}(0)$, 则存在 $g^{(n)} \in \mathcal{H}\left(g_{0}\right)$, 使得 $\varphi_{0}^{(n)}=\left(u_{0}^{(n)}, \omega_{0}^{(n)}\right)^{\mathrm{T}} \in \mathcal{A}_{\epsilon_{n}}^{g^{(n)}}(0)$. 令 $\varphi^{(n)}(t)=\left(u^{(n)}(t), \dot{u}^{(n)}(t)\right)^{\mathrm{T}}$ 是 $(2.9)$ 的解序列, 使得 $\varphi^{(n)}(0)$ $=\varphi_{0}^{(n)}$, 即

$$
\epsilon_{n} \ddot{u}^{(n)}+\dot{u}^{(n)}+A u^{(n)}+\lambda u^{(n)}+f\left(u^{(n)}\right)=g^{(n)}(t), \quad u^{(n)}(0)=u_{0}^{(n)}, \quad \dot{u}^{(n)}(0)=\omega_{0}^{(n)},
$$


那么

$$
\varphi^{(n)}(t)=\left(u^{(n)}(t), \dot{u}^{(n)}(t)\right)^{\mathrm{T}} \in \mathcal{A}_{\epsilon_{n}}^{g^{(n)}}(t), \quad \forall t \in \mathbb{R} .
$$

由 $\mathcal{H}\left(g_{0}\right)$ 在 $C_{b}\left(\mathbb{R}, \ell^{2}\right)$ 中的紧性知, 存在 $\left\{g^{(n)}\right\}_{n=1}^{+\infty}$ 的子列 (仍记作 $\left\{g^{(n)}\right\}_{n=1}^{+\infty}$ ), 使得

$$
g^{(n)} \rightarrow \bar{g} \in \mathcal{H}\left(g_{0}\right)(n \rightarrow+\infty) \text { 在 } C_{b}\left(\mathbb{R}, \ell^{2}\right) \text { 中强收敛. }
$$

接下来, 利用 Arzéla-Ascoli 定理和对角线序列法来证明 $\{n\}$ 存在子列 $\left\{n_{k}\right\}$ 使得

$$
\varphi^{\left(n_{k}\right)}(t)=\left(u^{\left(n_{k}\right)}(t), \dot{u}^{\left(n_{k}\right)}(t)\right)^{\mathrm{T}} \rightarrow(\bar{u}(t), \tilde{u}(t))^{\mathrm{T}} \in \mathcal{A}_{0}^{\bar{g}}(t)(k \rightarrow+\infty) \text { 在 } E \text { 中强收敛, } \quad \forall t \in \mathbb{R} \text {. }
$$

设 $J_{k}=[-k, k], k \in \mathbb{Z}_{+}$, 那么 $J_{k} \subset J_{k+1}$ 以及 $\bigcup_{k \in \mathbb{Z}_{+}} J_{k}=\mathbb{R}$. 由 (4.14), 可得

$$
\sup _{t \in \mathbb{R}} \sup _{n \in \mathbb{N}}\left\|\left(u^{(n)}(t), \dot{u}^{(n)}(t)\right)^{\mathrm{T}}\right\|_{\ell^{2} \times \ell^{2}} \leqslant \sqrt{2 M_{4}}, \quad \epsilon \sup _{t \in \mathbb{R}}\left\|\ddot{u}^{(n)}(t)\right\|^{2} \leqslant 2 M_{4} .
$$

取 $s_{1}, s_{2} \in J_{k}$, 由 (5.7), 有

$$
\left\|u^{(n)}\left(t_{1}\right)-u^{(n)}\left(t_{2}\right)\right\|=\left\|\dot{u}^{(n)}\left(t_{2}+\vartheta_{n}\left(t_{1}-t_{2}\right)\right)\right\| \cdot\left|t_{1}-t_{2}\right| \leqslant \sqrt{2 M_{4}}\left|t_{1}-t_{2}\right|, \quad\left|\vartheta_{n}\right| \leqslant 1,
$$

这表明 $\left\{u^{(n)}(t)\right\}$ 在 $C\left(J_{k}, \ell^{2}\right)$ 中是等度连续的.

由 (5.7) 可得, 存在 $\left\{\left(u^{(n)}(t), \dot{u}^{(n)}(t)\right)^{\mathrm{T}}\right\}$ 的子序列 (仍记作 $\left.\left\{\left(u^{(n)}(t), \dot{u}^{(n)}(t)\right)^{\mathrm{T}}\right\}\right)$ 和 $(\bar{u}(t), \tilde{u}(t))^{\mathrm{T}}$ $\in \ell^{2} \times \ell^{2}$ 使得

$$
\left(u^{(n)}(t), \dot{u}^{(n)}(t)\right)^{\mathrm{T}} \rightarrow(\bar{u}(t), \tilde{u}(t))^{\mathrm{T}}(n \rightarrow+\infty) \text { 在 } \ell^{2} \times \ell^{2} \text { 中弱收敛, } \forall t \in \mathbb{R}
$$

和

$$
\sup _{t \in \mathbb{R}}\left\|(\bar{u}(t), \tilde{u}(t))^{\mathrm{T}}\right\|_{\ell^{2} \times \ell^{2}}^{2} \leqslant 2 M_{4} .
$$

对于任意 $\eta>0$, 根据引理 4.2(iii) 可知, 存在 $I_{3}(\eta) \in \mathbb{N}$ 使得

$$
\sum_{\|m\| \geqslant I_{3}(\eta)}\left\|u_{m}^{(n)}(t)\right\|^{2} \leqslant \frac{\eta^{2}}{4}, \quad \forall t \in \mathbb{R} .
$$

令 $u^{(n)}(t)=w^{(n)}(t)+\varsigma^{(n)}(t)=\left(w_{m}^{(n)}(t)\right)_{m \in \mathbb{Z}^{K}}+\left(\varsigma_{m}^{(n)}(t)\right)_{m \in \mathbb{Z}^{K}}, t \in \mathbb{R}$, 其中,

$$
w_{m}^{(n)}(t)=\left\{\begin{array}{ll}
u_{m}^{(n)}(t), & \|m\| \leqslant I_{3}(\eta), \\
0, & \|m\|>I_{3}(\eta),
\end{array} \quad \varsigma_{m}^{(n)}(t)= \begin{cases}0, & \|m\| \leqslant I_{3}(\eta), \\
u_{m}^{(n)}(t), & \|m\|>I_{3}(\eta) .\end{cases}\right.
$$

由 (5.7) 和 (5.8) 得

$$
\left\|w^{(n)}(t)\right\|=\left(\sum_{\|m\| \leqslant I_{3}(\eta)}\left|u_{m}^{(n)}(t)\right|^{2}\right)^{\frac{1}{2}} \leqslant \sqrt{2 M_{4}}, \quad\left\|\varsigma^{(n)}(t)\right\|=\left(\sum_{\|m\|>I_{3}(\eta)}\left|u_{m}^{(n)}(t)\right|^{2}\right)^{\frac{1}{2}} \leqslant \frac{\eta}{2} .
$$

设

$$
\Gamma(t)=\left\{w(t)=\left(w_{m}(t)\right)_{\|m\| \leqslant I_{3}(\eta)} \in \mathbb{R}^{\left(2 I_{3}(\eta)+1\right)^{k}}: w_{m}(t) \in \mathbb{R},\left|w_{m}(t)\right| \leqslant \sqrt{2 M_{4}}\right\},
$$




$$
n_{\eta} \Gamma(t)=\left(\left[\frac{2 \sqrt{2 M_{4}} \sqrt{\left(2 I_{3}(\eta)+1\right)^{k}}}{\eta}\right]+1\right)^{\left(2 I_{3}(\eta)+1\right)^{k}} .
$$

那么 $\Gamma(t) \subset \mathbb{R}^{\left(2 I_{3}(\eta)+1\right)^{k}}$ 可以由 $n_{\eta} \Gamma(t)$ 个半径为 $\frac{\eta}{2}$ 、中心在 $w^{(l)}(t)=\left(w_{m}^{(l)}(t)\right)_{\|m\| \leqslant I_{3}(\eta)} \in \Gamma(t)(1 \leqslant l$ $\left.\leqslant n_{\eta} \Gamma(t)\right)$ 的闭球覆盖. 对于每一个 $1 \leqslant l \leqslant n_{\eta} \Gamma(t)$, 设 $\nu^{(l)}(t)=\left(\nu_{m}^{(l)}(t)\right)_{m \in \mathbb{Z}^{k}} \in \ell^{2}$, 使得

$$
\nu_{m}^{(l)}(t)= \begin{cases}w_{m}^{(l)}(t), & \|m\| \leqslant I_{3}(\eta), \\ 0, & \|m\|>I_{3}(\eta) .\end{cases}
$$

于是, 对于任意 $w^{(n)}(t) \in \Gamma(t)$, 存在 $l_{0} \in\left\{1,2, \ldots, n_{\eta} \Gamma(t)\right\}$ 使得

$$
\left\|w^{(n)}(t)-\nu^{\left(l_{0}\right)}(t)\right\|=\left(\sum_{\|m\| \leqslant I_{3}(\eta)}\left|u_{m}^{(n)}(t)-w_{m}^{\left(l_{0}\right)}(t)\right|^{2}\right)^{\frac{1}{2}} \leqslant \frac{\eta}{2} .
$$

由 (5.9) 和 (5.10), 有

$$
\left\|u^{(n)}(t)-\nu^{\left(l_{0}\right)}(t)\right\|=\left\|w^{(n)}(t)-\nu^{\left(l_{0}\right)}(t)+\varsigma^{(n)}(t)\right\| \leqslant\left\|w^{(n)}(t)-\nu^{\left(l_{0}\right)}(t)\right\|+\left\|\varsigma^{(n)}(t)\right\| \leqslant \eta .
$$

因此 $\left\{u^{(n)}(t)\right\}_{n=1}^{\infty}$ 可以由 $n_{\eta} \Gamma(t)$ 个半径为 $\eta$ 、中心在 $\left\{\nu^{\left(l_{0}\right)}(t): 1 \leqslant l_{0} \leqslant n_{\eta} \Gamma(t)\right\}$ 的闭球覆盖. 因此 $\left\{u^{(n)}(t)\right\}_{n=1}^{\infty}$ 在 $\ell^{2}$ 中是预紧的, 即 $\left\{u^{(n)}(t)\right\}_{n=1}^{\infty}$ 在 $\ell^{2}$ 中有强收玫于 $\bar{u}(t)$ 的子列 $\left\{u^{\left(n_{k}\right)}(t)\right\}_{k=1}^{\infty}$.

由 Arzéla-Ascoli 定理知, $\left\{u^{(n)}(t)\right\}$ 有一个子列 $\left\{u^{(n, 1)}(t)\right\}_{n=1}^{+\infty}$ 使得

$$
u^{(n, 1)}(t) \rightarrow \bar{u}(t)(n \rightarrow+\infty) \text { 在 } C\left(J_{1}, \ell^{2}\right) \text { 中强收敛. }
$$

再次使用 Arzéla-Ascoli 定理以及重复上面的方法, 可得对于任意 $k \in \mathbb{N},\left\{u^{(n, k)}(t)\right\}$ 有子列 $\left\{u^{(n, k+1)}(t)\right\}$ 使得

$$
u^{(n, k+1)}(t) \rightarrow \bar{u}(t)(n \rightarrow+\infty) \text { 在 } C\left(J_{k+1}, \ell^{2}\right) \text { 中强收敛. }
$$

取 $\left\{u^{(n, k)}(t)\right\}$ 的对角线序列, 可得存在子序列 $\left\{u^{(k, k)}(t)\right\}=\left\{u^{\left(k_{k}\right)}(t)\right\}\left(\epsilon_{n}\right.$ 有对应的子列 $\left.\epsilon_{\left(k_{k}\right)}\right)$, 使得对 于任意紧子集 $J \subset \mathbb{R}$ (包含在某个闭区间 $J_{k}$ 中), 有

$$
u^{\left(k_{k}\right)}(t) \rightarrow \bar{u}(t)(k \rightarrow+\infty) \text { 在 } C\left(J, \ell^{2}\right) \text { 中强收敛. }
$$

由 (5.5) 得

$$
\dot{u}^{\left(k_{k}\right)}(t)=-\epsilon_{\left(k_{k}\right)} \ddot{\bar{u}}^{\left(k_{k}\right)}(t)-A u^{\left(k_{k}\right)}(t)-\lambda u^{\left(k_{k}\right)}(t)-f\left(u^{\left(k_{k}\right)}(t)\right)+g^{\left(k_{k}\right)}(t), \quad t \in \mathbb{R} .
$$

由 (5.7) 和 $0<\epsilon_{k_{k}} \rightarrow 0^{+}(k \rightarrow+\infty)$, 有

$$
\sup _{t \in \mathbb{R}} \sqrt{\epsilon_{k_{k}}}\left\|\ddot{\bar{u}}^{\left(k_{k}\right)}(t)\right\| \leqslant \sqrt{2 M_{4}}<\infty, \quad \lim _{n \rightarrow+\infty} \sup _{t \in \mathbb{R}}\left(\epsilon_{k_{k}}\left\|\ddot{\bar{u}}^{\left(k_{k}\right)}(t)\right\|\right)=0 .
$$

由 (5.6)、(5.12)-(5.13) 和 $f$ 的连续性知, 对于任意紧子集 $J \subset \mathbb{R}$, 有

$$
\dot{u}^{\left(k_{k}\right)(t)} \rightarrow-A \bar{u}(t)-\lambda \bar{u}(t)-f(\bar{u}(t))+\bar{g}(t)(k \rightarrow+\infty) \text { 在 } C\left(J, \ell^{2}\right) \text { 中强收敛, }
$$

其中 $-A \bar{u}(t)-\lambda \bar{u}(t)-f(\bar{u}(t))+\bar{g}(t) \in C_{b}\left(\mathbb{R}, \ell^{2}\right)$. 由极限的唯一性可得

$$
\tilde{u}(t)=\dot{\bar{u}}(t)=-A \bar{u}(t)-\lambda \bar{u}(t)-f(\bar{u}(t))+\bar{g}(t) \in C_{b}\left(\mathbb{R}, \ell^{2}\right) .
$$


因此, 对于任意 $t \in \mathbb{R}$, 有 $\bar{u}(t) \in \mathcal{A}^{\bar{g}}(t)$. 故对于任意 $t \in \mathbb{R}$, 有 $(\bar{u}(t), \dot{\bar{u}}(t))^{\mathrm{T}} \in \mathcal{A}_{0}^{\bar{g}}(t)$. 由 (5.11) 和 (5.14), 有

$$
\left(u^{\left(k_{k}\right)}(t), \dot{u}^{\left(k_{k}\right)}(t)\right)^{\mathrm{T}} \rightarrow(\bar{u}(t), \tilde{u}(t))^{\mathrm{T}}(k \rightarrow+\infty) \text { 在 } C(J, E) \text { 中强收敛. }
$$

特别地, 当 $k \rightarrow+\infty$ 时, 有

$$
\varphi_{0}^{\left(k_{k}\right)}=\left(u_{0}^{\left(k_{k}\right)}, \omega_{0}^{\left(k_{k}\right)}\right)^{\mathrm{T}}=\left(u^{\left(k_{k}\right)}(0), \dot{u}^{\left(k_{k}\right)}(0)\right)^{\mathrm{T}} \rightarrow(\bar{u}(0), \tilde{u}(0))^{\mathrm{T}} \in \mathcal{A}_{0}^{\bar{g}}(0) \subseteq \mathcal{A}_{0}^{\mathcal{H}\left(g_{0}\right)} \text { 在 } E \text { 中强收敛. }
$$

证毕.

定理 5.1 假设条件 (A1)-(A5) 成立, 则

$$
\lim _{\epsilon \rightarrow 0^{+}} d_{h}\left(A_{\epsilon}^{\mathcal{H}\left(g_{0}\right)}, A_{0}^{\mathcal{H}\left(g_{0}\right)}\right)=0, \quad \lim _{\epsilon \rightarrow 0^{+}} d_{h}\left(\Pi_{1} A_{\epsilon}^{\mathcal{H}\left(g_{0}\right)}, A_{0}^{\mathcal{H}\left(g_{0}\right)}\right)=0, \quad \forall t \in \mathbb{R} .
$$

证明 假设 $\lim _{\epsilon \rightarrow 0^{+}} d_{h}\left(\mathcal{A}_{\epsilon}^{\mathcal{H}\left(g_{0}\right)}, \mathcal{A}_{0}^{\mathcal{H}\left(g_{0}\right)}\right) \neq 0$, 那么存在 $\eta_{0}>0 、\left\{\epsilon_{n}\right\}_{n=1}^{+\infty} \subset(0, \bar{\epsilon}] 、 \epsilon_{n} \rightarrow 0(n \rightarrow+\infty)$ 和 $\left(u^{(n)}, v^{(n)}\right)^{\mathrm{T}} \in \mathcal{A}_{\epsilon_{n}}^{\mathcal{H}\left(g_{0}\right)}$ 使得

$$
d_{h}\left(\left(u^{(n)}, v^{(n)}\right)^{\mathrm{T}}, \mathcal{A}_{0}^{\mathcal{H}\left(g_{0}\right)}\right) \geqslant \eta_{0}, \quad n \in \mathbb{Z}_{+} .
$$

根据引理 5.1 可得 $\left\{\left(u^{(n)}, v^{(n)}\right)^{\mathrm{T}}\right\}_{n \in \mathbb{Z}_{+}}$有收玫于 $\mathcal{A}_{0}^{\mathcal{H}\left(g_{0}\right)}$ 中元素的子列, 与 (5.17) 相矛盾. 证毕.

\section{6 一致吸引子在正二阶项系数处的上半连续性}

设 $\epsilon_{0}>0$ 是一个正常数. 本节讨论当 $\epsilon \rightarrow \epsilon_{0}$ 时系统 $(2.9)$ 的一致吸引子 $\mathcal{A}_{\epsilon}^{\mathcal{H}\left(g_{0}\right)}$ 的上半连续性.

记 $\wedge=\left[\frac{\epsilon_{0}}{2}, \frac{3 \epsilon_{0}}{2}\right]$. 考虑 $(2.9)$ 的一致吸引子簇 $\left\{\mathcal{A}_{\epsilon}^{\mathcal{H}\left(g_{0}\right)}\right\}_{\epsilon \in \wedge}$.

引理 6.1 假设 (A1)-(A5) 成立, $\left\{V_{\epsilon}^{g}(t, \tau)\right\}_{t \geqslant \tau, \epsilon \in \wedge, g \in \mathcal{H}\left(g_{0}\right)}$ 是 (3.33) 定义在 $E$ 上的连续过程 簇, 则

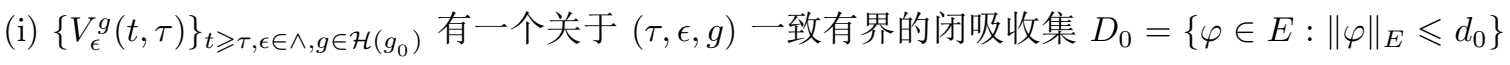
$\left(d_{0}>0\right.$ 独立于 $\left.(\tau, \epsilon, g)\right)$;

(ii) $\forall \epsilon \in \wedge,\left\{V_{\epsilon}^{g}(t, \tau)\right\}_{t \geqslant \tau, g \in \mathcal{H}\left(g_{0}\right)}$ 有一致吸引子 $A_{\epsilon}^{\mathcal{H}\left(g_{0}\right)} \subseteq D_{0} \subset E$;

(iii) $\bigcup_{\epsilon \in \wedge} A_{\epsilon}^{\mathcal{H}\left(g_{0}\right)}$ 在 $E$ 中是预紧的.

证明 (i) 由 (3.34), 可得

$$
\tilde{r}_{\epsilon}=\frac{2}{\sqrt{\epsilon}} \sqrt{\frac{\delta_{2}}{\mu}\left\|g_{0}\right\|^{2}+\frac{\lambda_{0} \delta_{2}}{\mu}\|b\|^{2}} \leqslant \frac{2 \sqrt{2}}{\sqrt{\epsilon_{0}}} \sqrt{\frac{\delta_{5}}{\tilde{\mu}}\left\|g_{0}\right\|^{2}+\frac{\lambda_{0} \delta_{5}}{\tilde{\mu}}\|b\|^{2}}=d_{0}, \quad \forall \epsilon \in \wedge,
$$

其中,

$$
\delta_{5}=2+3 \epsilon_{0} \lambda_{0}+\frac{3 \epsilon_{0}}{2 \lambda_{0}} \geqslant \delta_{2}, \quad \tilde{\mu}=\frac{\lambda_{0}}{2 \sqrt{1+6 \epsilon_{0} \lambda_{0}}\left(1+\sqrt{1+6 \epsilon_{0} \lambda_{0}}\right)} \leqslant \mu .
$$

根据定理 3.1(ii) 和引理 3.2 的证明可知, 过程簇 $\left\{V_{\epsilon}^{g}(t, \tau)\right\}_{t \geqslant \tau, \epsilon \in \wedge, g \in \mathcal{H}\left(g_{0}\right)}$ 存在有界闭吸收集

$$
D_{0}=\left\{\varphi=(u, \omega)^{\mathrm{T}} \in E:\|\varphi\|_{E}=\sqrt{\|u\|^{2}+\|\omega\|^{2}} \leqslant d_{0}\right\}
$$

(独立于 $(\tau, \epsilon, g))$ 使得存在 $T\left(D_{0}\right)>0$ (独立于 $\left.(\epsilon, g)\right)$ 使得

$$
V_{\epsilon}^{g}(t, t-s) D_{0} \subseteq D_{0}, \quad \forall t \in \mathbb{R}, \quad s \geqslant T\left(D_{0}\right), \quad \epsilon \in \wedge, \quad g \in \mathcal{H}\left(g_{0}\right) .
$$


(ii) 由定理 3.1(v) 可知, 对于任意 $\epsilon \in \wedge,\left\{V_{\epsilon}^{g}(t, \tau)\right\}_{t \geqslant \tau, g \in \mathcal{H}\left(g_{0}\right)}$ 存在紧的一致吸引子 $\mathcal{A}_{\epsilon}^{\mathcal{H}\left(g_{0}\right)} \subseteq D_{0}$. 因此,

$$
\bigcup_{\epsilon \in \wedge} \mathcal{A}_{\epsilon}^{\mathcal{H}\left(g_{0}\right)} \subseteq D_{0} \subset E
$$

(iii) 只需证明: 对于任意 $\eta>0, \bigcup_{\epsilon \in \wedge} \mathcal{A}_{\epsilon}^{\mathcal{H}\left(g_{0}\right)}$ 在 $E$ 上可以由有限个半径为 $\eta$ 的球所覆盖.

设

$$
\hat{\psi}=(\hat{u}, \hat{v})^{\mathrm{T}} \in \bigcup_{\epsilon \in \wedge} \mathcal{A}_{\epsilon}^{\mathcal{H}\left(g_{0}\right)}=\bigcup_{\epsilon \in \wedge} \bigcup_{g \in \mathcal{H}\left(g_{0}\right)} \mathcal{A}_{\epsilon}^{g}(0) \subseteq D_{0} \subset E
$$

那么存在 $\hat{g} \in \mathcal{H}\left(g_{0}\right)$ 和 $\hat{\epsilon} \in \Lambda$ 使得 $\hat{\psi} \in \mathcal{A}_{\hat{\epsilon}}^{\hat{g}}(0)$. 令 $\varphi_{\hat{\epsilon}, \hat{g}}(t)=\left(u_{\hat{\epsilon}, \hat{g}}(t), \dot{u}_{\hat{\epsilon}, \hat{g}}(t)\right)^{\mathrm{T}}$ 是问题

$$
\hat{\epsilon} \ddot{u}_{\hat{\epsilon}, \hat{g}}+\dot{u}_{\hat{\epsilon}, \hat{g}}+A u_{\hat{\epsilon}, \hat{g}}+\lambda u_{\hat{\epsilon}, \hat{g}}+f\left(u_{\hat{\epsilon}, \hat{g}}\right)=\hat{g}(t), \quad u_{\hat{\epsilon}, \hat{g}}(0)=\hat{u}, \quad \dot{u}_{\hat{\epsilon}, \hat{g}}(0)=\hat{v}
$$

的解, 那么

$$
\begin{aligned}
& \varphi_{\hat{\epsilon}, \hat{g}}(0)=\hat{\psi}=(\hat{u}, \hat{v})^{\mathrm{T}}, \quad \varphi_{\hat{\epsilon}, \hat{g}}(t)=\left(u_{\hat{\epsilon}, \hat{g}}(t), \dot{u}_{\hat{\epsilon}, \hat{g}}(t)\right)^{\mathrm{T}} \in \mathcal{A}_{\hat{\epsilon}}^{\hat{g}}(t) \subseteq D_{0}, \quad \forall t \in \mathbb{R}, \\
& \left\|\varphi_{\hat{\epsilon}, \hat{g}}(t)\right\|_{E}^{2}=\left\|u_{\hat{\epsilon}, \hat{g}}(t)\right\|^{2}+\left\|\dot{u}_{\hat{\epsilon}, \hat{g}}(t)\right\|^{2} \leqslant d_{0}^{2}, \quad \forall t \in \mathbb{R} .
\end{aligned}
$$

令

$$
\psi_{\hat{\epsilon}, \hat{g}}(t)=\psi_{\hat{\epsilon}, \hat{g}}(t, t-s)=\left(u_{\hat{\epsilon}, \hat{g}}(t), \dot{u}_{\hat{\epsilon}, \hat{g}}(t)+\frac{\hat{\delta}}{\hat{\epsilon}} u_{\hat{\epsilon}, \hat{g}}(t)\right)^{\mathrm{T}}=L_{\epsilon} \varphi_{\hat{\epsilon}, \hat{g}}(t) \in \mathcal{K}_{\hat{\epsilon}}^{\hat{g}}(t), \quad s \geqslant 0,
$$

其中 $\hat{\delta}=\frac{\hat{\epsilon} \lambda_{0}}{1+4 \hat{\epsilon} \lambda_{0}}$. 类似于引理 3.2 , 由 (6.7), 有

$$
\begin{aligned}
& \frac{d}{d t}\left[\sum_{m \in \mathbb{Z}^{k}} \theta\left(\frac{\|m\|}{K}\right)\left|\psi_{\hat{\epsilon}, \hat{g}, m}(t)\right|_{E_{\lambda, \epsilon}}^{2}+\frac{2}{\epsilon} \sum_{m \in \mathbb{Z}^{k}} \theta\left(\frac{\|m\|}{K}\right)\left(G_{m}\left(u_{\hat{\epsilon}, \hat{g}, j}(t) \mid j \in I_{m q}\right)+b_{m}^{2}\right)\right] \\
& +\tilde{\mu}\left[\sum_{m \in \mathbb{Z}^{k}} \theta\left(\frac{\|m\|}{K}\right)\left|\psi_{\hat{\epsilon}, \hat{g}, m}(t)\right|_{E_{\lambda, \epsilon}}^{2}+\frac{2}{\epsilon} \sum_{m \in \mathbb{Z}^{k}} \theta\left(\frac{\|m\|}{K}\right)\left(G_{m}\left(u_{\hat{\epsilon}, \hat{g}, j}(t) \mid j \in I_{m q}\right)+b_{m}^{2}\right)\right] \\
& \quad \leqslant \frac{1}{\epsilon}\left(\frac{\delta_{6}}{K}+2 \sum_{\|m\| \geqslant K} \hat{g}_{m}^{2}(t)+2 \lambda_{0} \sum_{\|m\| \geqslant K} b_{m}^{2}\right),
\end{aligned}
$$

其中,

$$
\begin{aligned}
& \delta_{6}=k c_{1} d_{0}^{2}\left(8+2 \lambda_{0}+4 \lambda_{0}^{2}\right)+\frac{C_{0} q d_{0}^{2}}{4} \\
& \left|\psi_{\hat{\epsilon}, \hat{g}, m}\right|_{E_{\lambda, \hat{\epsilon}}}^{2}=\hat{\epsilon}^{-1} \sum_{j=1}^{k}\left(B_{j} u_{\hat{\epsilon}, \hat{g}}\right)_{m}^{2}+\hat{\epsilon}^{-1} \lambda_{m} u_{\hat{\epsilon}, \hat{g}, m}^{2}+\left(\dot{u}_{\hat{\epsilon}, \hat{g}, m}+\frac{\hat{\delta}}{\hat{\epsilon}} u_{\hat{\epsilon}, \hat{g}, m}\right)^{2} .
\end{aligned}
$$

类似于 (4.18)-(4.20) 可得, 对于任意 $\eta>0$, 存在 $T_{3}\left(\eta, d_{0}\right) \geqslant 0$ 和 $I_{4}(\eta) \in \mathbb{N}$ (独立于 $(t, \epsilon, g) \in \mathbb{R} \times \wedge$ $\left.\times \mathcal{H}\left(g_{0}\right)\right)$ 使得

$$
\sum_{\|m\| \geqslant 2 K}\left|\psi_{\hat{\epsilon}, \hat{g}, m}(t, t-s)\right|_{E_{\lambda, \hat{\epsilon}}}^{2} \leqslant \frac{\eta^{2}}{\delta_{5}}, \quad \forall t \in \mathbb{R}, \quad K \geqslant I_{4}(\eta), \quad s \geqslant T_{3}\left(\eta, d_{0}\right) .
$$


此外

$$
\begin{aligned}
\left|\varphi_{\hat{\epsilon}, \hat{g}, m}\right|_{E}^{2} & =u_{\hat{\epsilon}, \hat{g}, m}^{2}+\dot{u}_{\hat{\epsilon}, \hat{g}, m}^{2} \\
& \leqslant \delta_{5}\left[\hat{\epsilon}^{-1} \sum_{j=1}^{k}\left(B_{j} u_{\hat{\epsilon}, \hat{g}}\right)_{m}^{2}+\hat{\epsilon}^{-1} \lambda_{m} u_{\hat{\epsilon}, \hat{g}, m}^{2}+\left(\dot{u}_{\hat{\epsilon}, \hat{g}, m}+\frac{\hat{\delta}}{\hat{\epsilon}} u_{\hat{\epsilon}, \hat{g}, m}\right)^{2}\right] \\
& =\delta_{5}\left|\psi_{\hat{\epsilon}, \hat{g}, m}\right|_{E_{\lambda, \epsilon}}^{2} .
\end{aligned}
$$

因此

$$
\sum_{\|m\| \geqslant 2 K}\left|\varphi_{\hat{\epsilon}, \hat{g}, m}(t, t-s)\right|_{E}^{2} \leqslant \eta^{2}, \quad \forall t \in \mathbb{R}, \quad K \geqslant I_{4}(\eta), \quad s \geqslant T_{3}\left(\eta, d_{0}\right) .
$$

特别地, 对于任意 $\eta>0$, 有

$$
\sum_{\|m\| \geqslant 2 I_{4}(\eta)}\left|\psi_{m}\right|_{E}^{2}=\sum_{\|m\| \geqslant 2 I_{4}(\eta)}\left|\varphi_{\hat{\epsilon}, \hat{g}, m}(0)\right|_{E}^{2}=\sum_{\|m\| \geqslant 2 I_{4}(\eta)}\left|\varphi_{\hat{\epsilon}, \hat{g}, m}\left(0,-T_{3}\left(\eta, d_{0}\right)\right)\right|_{E}^{2} \leqslant \eta^{2} .
$$

所以, 由 $E=\ell^{2} \times \ell^{2}$ 中预紧集的特征可知, $\bigcup_{\epsilon \in \wedge} \mathcal{A}_{\epsilon}^{\mathcal{H}\left(g_{0}\right)}$ 在 $E$ 中是预紧的. 证毕.

当 $\epsilon=\epsilon_{0}>0$ 时, $(2.9)$ 为

$$
\left\{\begin{array}{l}
\epsilon_{0} \ddot{u}+\dot{u}+A u+\lambda u+f(u)=g(t), \quad t>\tau, \quad \epsilon_{0}>0, \quad g \in \mathcal{H}\left(g_{0}\right) . \\
u(\tau)=u_{\tau}, \quad \dot{u}(\tau)=u_{1 \tau},
\end{array}\right.
$$

由第 3 节知, (6.11) 的解生成 $E$ 上的过程簇 $\left\{V_{\epsilon_{0}}^{g}(t, \tau)\right\}_{t \geqslant \tau, g \in \mathcal{H}\left(g_{0}\right)}$, 并且存在吸收集 $D_{0}$ 和一致吸 引子 $\mathcal{A}_{\epsilon_{0}}^{\mathcal{H}\left(g_{0}\right)}$.

引理 6.2 假设条件 (A1)-(A5) 成立. 取任意正实数列 $\left\{\epsilon_{n}\right\}_{n=1}^{+\infty} \subset \wedge$, 且 $\epsilon_{n} \rightarrow \epsilon_{0}(n \rightarrow+\infty)$. 设 $\breve{\varphi}^{(n)}=\left(\breve{u}^{(n)}, \breve{v}^{(n)}\right)^{\mathrm{T}} \in A_{\epsilon_{n}}^{\mathcal{H}\left(g_{0}\right)}$, 那么存在 $\{n\}$ 的子列 $\left\{n_{k}\right\}$ 和 $(\breve{u}, \breve{v})^{\mathrm{T}} \in A_{\epsilon_{0}}^{\mathcal{H}\left(g_{0}\right)}$, 使得

$$
\breve{\varphi}^{\left(n_{k}\right)}=\left(\breve{u}^{\left(n_{k}\right)}, \breve{v}^{\left(n_{k}\right)}\right)^{\mathrm{T}} \rightarrow(\breve{u}, \breve{v})^{\mathrm{T}} \in \mathcal{A}_{\epsilon_{0}}^{\mathcal{H}\left(g_{0}\right)}(k \rightarrow+\infty) \text { 在 } E \text { 中强收敛. }
$$

证明 因为 $t \in \mathbb{R}, \breve{\varphi}^{(n)}=\left(\breve{u}^{(n)}, \breve{v}^{(n)}\right)^{\mathrm{T}} \in \mathcal{A}_{\epsilon_{n}}^{\mathcal{H}\left(g_{0}\right)}=\bigcup_{g \in \mathcal{H}\left(g_{0}\right)} \mathcal{A}_{\epsilon_{n}}^{g}(0)=\bigcup_{g \in \mathcal{H}\left(g_{0}\right)} \mathcal{A}_{\epsilon_{n}}^{g}(t)$, 所以存在 $g_{n} \in \mathcal{H}\left(g_{0}\right)$ 使得 $\breve{\varphi}^{(n)}=\left(\breve{u}^{(n)}, \breve{v}^{(n)}\right)^{\mathrm{T}} \in \mathcal{A}_{\epsilon_{n}}^{g_{n}}(0)$. 令 $\varphi^{(n)}(t)=\varphi^{(n)}(t, t-s)=\left(u^{(n)}(t), \dot{u}^{(n)}(t)\right)^{\mathrm{T}}(s \geqslant 0)$ 是 $(2.9)$ 的解序列, 使得 $\varphi^{(n)}(0)=\breve{\varphi}^{(n)}$, 即

$$
\epsilon_{n} \ddot{u}^{(n)}+\dot{u}^{(n)}+A u^{(n)}+\lambda u^{(n)}+f\left(u^{(n)}\right)=g_{n}(t), \quad u^{(n)}(0)=\breve{u}^{(n)}, \quad \dot{u}^{(n)}(0)=\breve{v}^{(n)},
$$

那么

$$
\varphi^{(n)}(t)=\left(u^{(n)}(t), \dot{u}^{(n)}(t)\right)^{\mathrm{T}} \in \mathcal{A}_{\epsilon_{n}}^{g_{n}}(t) \subseteq D_{0} \subset E, \quad \forall t \in \mathbb{R} .
$$

类似于引理 4.2 的证明, 存在正常数 $M_{5}=M_{5}\left(\epsilon_{0}\right)>0$ (独立于 $\left.\left(g_{n}, \epsilon_{n}\right)\right)$, 使得

$$
\sup _{n \in \mathbb{N}} \sup _{t \in \mathbb{R}}\left(\left\|\ddot{u}^{(n)}(t)\right\|^{2}+\left\|\dot{u}^{(n)}(t)\right\|^{2}+\left\|u^{(n)}(t)\right\|^{2}\right) \leqslant M_{5}, \quad \sup _{n \in \mathbb{N}} \sup _{t \in \mathbb{R}}\left\|\dot{\varphi}^{(n)}(t)\right\|_{E}^{2} \leqslant M_{5},
$$

以及对于任意 $\eta>0$, 存在 $I_{5}(\eta) \in \mathbb{N}$ (独立于 $\left(g_{n}, \epsilon_{n}\right)$ ) 使得对于 $K \geqslant I_{5}(\eta)$, 有

$$
\sup _{n \in \mathbb{N}} \sup _{t \in \mathbb{R}} \sum_{\|m\| \geqslant 2 I_{5}(\eta)}\left(\left|u_{m}^{(n)}(t)\right|^{2}+\left|\dot{u}_{m}^{(n)}(t)\right|^{2}\right) \leqslant \frac{\eta^{2}}{4} .
$$


由 $\mathcal{H}\left(g_{0}\right)$ 在 $C_{b}\left(\mathbb{R}, \ell^{2}\right)$ 中的紧性知, 存在 $\left\{g_{n}\right\}_{n=1}^{+\infty}$ 的子序列 (仍记作 $\left.\left\{g_{n}\right\}_{n=1}^{+\infty}\right)$ 和 $\tilde{g} \in \mathcal{H}\left(g_{0}\right)$ 使得

$$
g_{n} \rightarrow \tilde{g} \in \mathcal{H}\left(g_{0}\right)(n \rightarrow+\infty) \text { 在 } C_{b}\left(\mathbb{R}, \ell^{2}\right) \text { 中强收敛. }
$$

由 $\left\{\breve{\varphi}^{(n)}\right\}_{n=1}^{+\infty}=\left\{\left(\breve{u}^{(n)}, \breve{v}^{(n)}\right)^{\mathrm{T}}\right\}_{n=1}^{+\infty} \subset \bigcup_{\epsilon \in \wedge} \mathcal{A}_{\epsilon}^{\mathcal{H}\left(g_{0}\right)}$ 和引理 6.1(iii) 知, $\left\{\breve{\varphi}^{(n)}\right\}_{n=1}^{+\infty}$ 有收敛子列 $\left\{\breve{\varphi}^{(n)}\right\}_{n=1}^{+\infty}$ (仍记作 $\left.\left\{\breve{\varphi}^{(n)}\right\}_{n=1}^{+\infty}\right)$ 和 $\breve{\varphi}^{0}=\left(\breve{u}^{0}, \breve{v}^{0}\right)^{\mathrm{T}} \in \bigcup_{\epsilon \in \wedge} \mathcal{A}_{\epsilon}^{\mathcal{H}\left(g_{0}\right)}$ 使得

$$
\breve{\varphi}^{(n)}=\left(\breve{u}^{(n)}, \breve{v}^{(n)}\right)^{\mathrm{T}} \rightarrow \breve{\varphi}^{0}=\left(\breve{u}^{0}, \breve{v}^{0}\right)^{\mathrm{T}}(n \rightarrow+\infty) \text { 在 } E \text { 中强收敛. }
$$

设 $J_{k}=[-k, k], k \in \mathbb{Z}_{+}$. 取 $s_{1}, s_{2} \in J_{k}$, 由 $(6.15)$, 有

$$
\left\|\varphi^{(n)}\left(t_{1}\right)-\varphi^{(n)}\left(t_{2}\right)\right\|=\left\|\dot{\varphi}^{(n)}\left(t_{2}+\vartheta_{n}\left(t_{1}-t_{2}\right)\right)\right\| \cdot\left|t_{1}-t_{2}\right| \leqslant \sqrt{M_{5}}\left|t_{1}-t_{2}\right|, \quad\left|\vartheta_{n}\right| \leqslant 1,
$$

这蕴涵着 $\left\{\varphi^{(n)}(t)\right\}$ 在 $C\left(J_{k}, E\right)$ 中是等度连续的.

类似于引理 5.1, 由 (6.16) 可得 $\left\{\varphi^{(n)}(t)\right\}_{n=1}^{\infty}$ 在 $E$ 中是预紧的. 因此, 利用 Arzéla-Ascoli 定理和 对角线序列法, 可得对于任意紧子集 $J \subset \mathbb{R}$, 存在 $\left\{n_{k}\right\}$ 的子序列 $\{n\}$, 使得

$$
\begin{aligned}
\varphi^{\left(n_{k}\right)}(t) & =\left(u^{\left(n_{k}\right)}(t), \dot{u}^{\left(n_{k}\right)}(t)\right)^{\mathrm{T}} \rightarrow \varphi^{(0)}(t) \\
& =\left(u^{(0)}(t), \dot{u}^{(0)}(t)\right)^{\mathrm{T}}(k \rightarrow+\infty) \text { 在 } C(J, E) \text { 中强收敛, } \quad \forall t \in \mathbb{R}
\end{aligned}
$$

和

$$
\sup _{t \in \mathbb{R}}\left\|\varphi^{(0)}(t)\right\|_{E}^{2}=\sup _{t \in \mathbb{R}}\left\|\left(u^{(0)}(t), \dot{u}^{(0)}(t)\right)^{\mathrm{T}}\right\|_{E}^{2} \leqslant M_{5} .
$$

由 (6.13) 得

$$
\ddot{u}^{\left(n_{k}\right)}(t)=\frac{1}{\epsilon_{n_{k}}}\left[-\dot{u}^{\left(n_{k}\right)}(t)-A u^{\left(n_{k}\right)}(t)-\lambda u^{\left(n_{k}\right)}(t)-f\left(u^{\left(n_{k}\right)}(t)\right)+g_{n_{k}}(t)\right], \quad t \in \mathbb{R} .
$$

由 (6.17)、(6.19)、 $\epsilon_{k_{k}} \rightarrow \epsilon_{0}(k \rightarrow+\infty)$ 和 $f$ 的连续性可得, 当 $k \rightarrow+\infty$ 时, 对于任意紧子集 $J \subset \mathbb{R}$ 和 任意 $t \in \mathbb{R}$, 有

$$
\ddot{u}^{\left(n_{k}\right)}(t) \rightarrow \ddot{u}^{(0)}(t)=\frac{1}{\epsilon_{0}}\left[-\dot{u}^{(0)}(t)-A u^{(0)}(t)-\lambda u^{(0)}(t)-f\left(u^{(0)}(t)\right)+\tilde{g}(t)\right] \text { 在 } C(J, E) \text { 中强收敛, }
$$

即

$$
\epsilon_{0} \ddot{u}^{(0)}(t)+\dot{u}^{(0)}(t)+A u^{(0)}(t)+\lambda u^{(0)}(t)+f\left(u^{(0)}(t)\right)=\tilde{g}(t), \quad \forall t \in \mathbb{R},
$$

由 (6.18) 得

$$
\varphi^{(0)}(0)=\left(u^{(0)}(0), \dot{u}^{(0)}(0)\right)^{\mathrm{T}}=\lim _{k \rightarrow \infty}\left(u^{\left(n_{k}\right)}(0), \dot{u}^{\left(n_{k}\right)}(0)\right)^{\mathrm{T}}=\left(\breve{u}^{0}, \breve{v}^{0}\right)^{\mathrm{T}}=\breve{\varphi}^{0}
$$

由 (6.20)、(6.23) 和 (6.24) 可知, 对于任意 $t \in \mathbb{R}$, 有 $\varphi^{(0)}(t) \in \mathcal{A}_{\epsilon_{0}}^{\tilde{g}}(t)$. 特别地, $\breve{\varphi}^{\left(n_{k}\right)}(0)=\left(\breve{u}^{\left(n_{k}\right)}(0)\right.$, $\left.\breve{v}^{\left(n_{k}\right)}(0)\right)^{\mathrm{T}} \rightarrow \varphi^{(0)}(0)=\breve{\varphi}^{0} \in \mathcal{A}_{\epsilon_{0}}^{\tilde{g}}(0)$. 因此, (6.19) 蕴涵着 (6.12) 成立. 证毕.

定理 6.1 假设条件 $(\mathrm{A} 1)-(\mathrm{A} 5)$ 成立, $\epsilon_{0}>0$, 则 $\lim _{\epsilon \rightarrow \epsilon_{0}} d_{h}\left(A_{\epsilon}^{\mathcal{H}\left(g_{0}\right)}, A_{\epsilon_{0}}^{\mathcal{H}\left(g_{0}\right)}\right)=0$. 
证明 设 $\lim _{\epsilon \rightarrow \epsilon_{0}} d_{h}\left(\mathcal{A}_{\epsilon}^{\mathcal{H}\left(g_{0}\right)}, \mathcal{A}_{\epsilon_{0}}^{\mathcal{H}\left(g_{0}\right)}\right) \neq 0$, 那么存在 $\delta_{0}>0$ 、 $\left\{\epsilon_{n}\right\}_{n=1}^{+\infty} \subset \wedge 、 \epsilon_{n} \rightarrow \epsilon_{0}(n \rightarrow+\infty)$ 以 及 $\check{\varphi}_{\epsilon_{n}}=\left(\check{u}_{\epsilon_{n}}, \check{v}_{\epsilon_{n}}\right)^{\mathrm{T}} \in \mathcal{A}_{\epsilon_{n}}^{\mathcal{H}\left(g_{0}\right)}$, 使得

$$
d_{h}\left(\breve{\varphi}_{\epsilon_{n}}, \mathcal{A}_{\epsilon_{0}}^{\mathcal{H}\left(g_{0}\right)}\right) \geqslant \delta_{0}>0
$$

由引理 6.2 可得, 在 $E$ 中, 存在 $\left\{\breve{\varphi}_{\epsilon_{n}}\right\}_{n=1}^{+\infty}$ 的子列 $\left\{\breve{\varphi}_{\epsilon_{n_{k}}}\right\}_{k=1}^{+\infty}$ 使得 $\breve{\varphi}_{\epsilon_{n_{k}}} \rightarrow \breve{\varphi}_{0} \in \mathcal{A}_{\epsilon_{0}}^{\mathcal{H}\left(g_{0}\right)}$, 与 $(6.25)$ 矛盾. 证毕.

\section{致谢 作者非常感谢评审专家提出的宝贵建议.}

\section{参考文献}

1 Carvalho A N, Langa J A, Robinson J C. Attractors for Infinite-Dimensional Non-Autonomous Dynamical Systems. New York: Springer, 2012

2 Chepyzhov V V, Vishik M I. Attractors for Equations of Mathematical Physics. Providence: Amer Math Soc, 2002

3 Bates P W, Lu K N, Wang B X. Attractors for lattice dynamical systems. Internat J Bifur Chaos Appl Sci Engrg, 2001, 11: 143-153

4 Abdallah A Y. Upper semicontinuity of the attractor for a second order lattice dynamical system. Discrete Contin Dyn Syst Ser B, 2005, 5: 899-916

5 Abdallah A Y. Long-time behavior for second order lattice dynamical systems. Acta Appl Math, 2009, 106: 47-59

6 Jia X L, Zhao C D, Yang X B. Global attractor and Kolmogorov entropy of three component reversible Gray-Scott model on infinite lattices. Appl Math Comput, 2012, 218: 9781-9789

7 Wang B X. Dynamics of systems on infinite lattices. J Differential Equations, 2006, 221: 224-245

8 Zhou S F. Attractors for second order lattice dynamical systems. J Differential Equations, 2002, 179: 605-624

9 Zhou S F. Attractors for first order dissipative lattice dynamical systems. Phys D, 2003, 178: 51-61

10 Zhou S F, Shi W. Attractors and dimension of dissipative lattice systems. J Differential Equations, 2006, 224: 172-204

11 Zhao C D, Zhou S F. Upper semicontinuity of attractors for lattice systems under singular perturbations. Nonlinear Anal, 2010, 72: 2149-2158

12 Oliveira J C, Pereira J M, Perla Menzala G. Attractors for second order periodic lattices with nonlinear damping. J Difference Equ Appl, 2008, 14: 899-921

13 Wang B X. Asymptotic behavior of non-autonomous lattice systems. J Math Anal Appl, 2007, 331: 121-136

14 Abdallah A Y. Uniform global attractors for first order non-autonomous lattice dynamical systems. Proc Amer Math Soc, 2010, 138: 3219-3228

15 Abdallah A Y. Attractors for second order lattice systems with almost periodic symbols in weighted spaces. J Math Anal Appl, 2016, 442: 761-781

16 Huang J W, Han X Y, Zhou S F. Uniform attractors for non-autonomous Klein-Gordon-Schrödinger lattice systems. Appl Math Mech (English Ed), 2009, 30: 1597-1607

17 Zhao C D, Zhou S F. Compact uniform attractors for dissipative lattice dynamical systems with delays. Discrete Contin Dyn Syst, 2008, 21: 643-663

18 Zhou S F, Zhao C D, Liao X Y. Compact uniform attractors for dissipative non-autonomous lattice dynamical systems. Commun Pure Appl Anal, 2007, 6: 1087-1111

19 Abdallah A Y, Wannan R T. Second order non-autonomous lattice systems and their uniform attractors. Commun Pure Appl Anal, 2019, 18: 1827-1846

20 Wang J H, Gu A H. Existence of backwards-compact pullback attractors for non-autonomous lattice dynamical systems. J Difference Equ Appl, 2016, 22: 1906-1911

21 Zhao C D, Xue G, Łukaszewicz G. Pullback attractors and invariant measures for discrete Klein-Gordon-Schrödinger equations. Discrete Contin Dyn Syst Ser B, 2018, 23: 4021-4044

22 Zhao X-Q, Zhou S F. Kernel sections for processes and nonautonomous lattice systems. Discrete Contin Dyn Syst Ser B, 2008, 9: 763-785

23 Lei N, Zhou S F. Upper semicontinuity of pullback attractors for non-autonomous lattice systems under singular perturbations. Discrete Contin Dyn Syst, 2021, 42: 73-108

24 Bates P W, Lu K N, Wang B X. Attractors of non-autonomous stochastic lattice systems in weighted spaces. Phys D, 2014, 289: $32-50$

25 Caraballo T, Morillas F, Valero J. Random attractors for stochastic lattice systems with non-Lipschitz nonlinearity. J Difference Equ Appl, 2011, 17: 161-184 
26 Caraballo T, Morillas F, Valero J. Attractors of stochastic lattice dynamical systems with a multiplicative noise and non-Lipschitz nonlinearities. J Differential Equations, 2012, 253: 667-693

27 Wang B X. Existence and upper semicontinuity of attractors for stochastic equations with deterministic nonautonomous terms. Stoch Dyn, 2014, 14: 1450009

28 Freitas M M, Kalita P, Langa J A. Continuity of non-autonomous attractors for hyperbolic perturbation of parabolic equations. J Differential Equations, 2018, 264: 1886-1945

\section{Upper semicontinuity of uniform attractors for non-autonomous lattice systems under singular perturbations}

\section{Na Lei \& Shengfan Zhou}

Abstract In this paper, we study the upper semicontinuity of the uniform attractors for the second order nonautonomous lattice systems driven almost periodic force with respect to the coefficient of the second order derivative term under Hausdorff semidistance. First, we present the existence of uniform attractors for the second order nonautonomous lattice systems and the corresponding first order nonautonomous lattice systems under certain conditions, respectively. Then we consider the relationship between these two uniform attractors when the coefficient of the second derivation term tends to zero. The method is to construct a new set in the phase space of the second order lattice systems such that the uniform attractor of the first order lattice system is naturally embedded into this set as the first component, and prove that the uniform attractors for the second order nonautonomous lattice system can enter any neighborhood of this set when the coefficient of the second derivation term is small enough. Finally, we discuss the upper semicontinuity of the uniform attractors of the second order nonautonomous lattice systems at any positive coefficient of the second derivative term.

Keywords nonautonomous lattice system, singular perturbations, uniform attractor, upper semicontinuity MSC(2020) 37L50, 35B40, 35Q55

doi: 10.1360/SCM-2021-0372 